\title{
P.I. CONTROL OF NONLINEAR OSCILLATIONS FOR A SYSTEM WITH DELAY
}

M. Akian , P.-A. Bliman , M. Sorine

$\mathbf{N}^{\circ} \mathbf{3 4 2 2}$

May 7, 1998

THÈME 4

apport

derecherche 



\title{
P.I. CONTROL OF NONLINEAR OSCILLATIONS FOR A SYSTEM WITH DELAY
}

\author{
M. Akian* , P.-A. Bliman ${ }^{\dagger}$, M. Sorine ${ }^{\ddagger}$ \\ Thème 4 - Simulation et optimisation \\ de systèmes complexes \\ Projets Sosso et Meta2 \\ Rapport de recherche $\mathrm{n}^{\circ} 3422$ - May 7, 1998 - 34 pages
}

\begin{abstract}
We study the periodic oscillations of a 1st order delayed linear system with relay output and proportional+integral feedback and describe the behavior of the general solutions of the closed loop. We present results on control of the oscillations and on rejection of the perturbations.

For the system under study, we first exhibit a countable set of periodic limit cycles. We show that in the particular case where only proportional control is used, any solution tends in finite time towards one of the limit cycles (whose determination depends on the initial conditions). All the cycles are orbitally unstable except one of them, the only slowly oscillating one.

Finally, we provide some well-posedness and ultimate boundedness results for a timevarying perturbed version of the system under study. The given estimates show that the proportional+integral feedback law permits to reject various parametric perturbations.
\end{abstract}

Key-words: control of oscillations, fuel-air ratio regulation, delay differential equations, slowly oscillating solutions, super-high-frequency oscillations, relay nonlinearity.

(Résumé : tsvp)

\footnotetext{
* email: Marianne.Akian@inria.fr

$\dagger$ email: Pierre-Alexandre.Bliman@inria.fr

$\ddagger$ email: Michel.Sorine@inria.fr
} 


\section{Contrôle proportionnel-intégral d'oscillations non-linéaires pour un système à retard}

Résumé : Nous étudions les oscillations périodiques d'un système linéaire du ler ordre avec retard et relais, bouclé par une loi de contre-réaction proportionnelle-intégrale, et nous décrivons le comportement des solutions du système bouclé. Nous présentons des résultats de contrôle des oscillations et de rejet des perturbations.

Pour le système étudié, nous exhibons tout d'abord un ensemble dénombrable de cycleslimites périodiques. Nous montrons que, dans le cas particulier où seul un contrôle proportionnel est utilisé, toute solution tend en temps fini vers l'un des cycles-limites (dont la détermination dépend des conditions initiales). Tous les cycles sont orbitalement instables, à l'exception du plus lent, qui est orbitalement asymptotiquement stable, et également "lentement oscillant".

Nous montrons enfin un résultat d'existence et d'unicité des solutions d'une version perturbée non-autonome du système étudié. Des estimations montrent que les lois de contreréaction proportionnelle-intégrale permettent de rejeter asymptotiquement des perturbations paramétriques variées.

Mots-clé : contrôle des oscillations, régulation de la combustion, équations différentielles à retard, solutions lentement oscillantes, oscillations de super-haute fréquence, relais. 


\section{Introduction}

We consider periodic oscillations of relay systems. In Automatic Control applications, the relay nonlinearity is used to describe a sensor or actuator behavior. Such a nonlinearity combined with stabilizing feedback loops often leads to limit cycles for the closed loop system.

We study here the behavior of a first order system controlled by a Proportional+Integral control law on the delayed output of a relay sensor. This class of control laws permits to adjust the amplitude and the period of the limit cycles [3]. The system under study is the following:

$$
\left\{\begin{array}{l}
\dot{x}(t)=-\frac{1}{\tau}\left(k_{I} y(t)+k_{P} \operatorname{sgn} x(t-h)+x(t)\right) \\
\dot{y}(t)=\operatorname{sgn} x(t-h)
\end{array}\right.
$$

where $\tau>0$ is the time constant of the plant, $h>0$ the delay, and $k_{I}, k_{P}$ the PI controller parameters. It is indeed obtained by closing the open-loop system (where $u$ is the control and $Y$ the output) $\tau \dot{x}+x=u, Y=\operatorname{sgn} x(t-h)$ with the P.I. control law $u=-k_{P} Y-k_{I} \int Y$, in an attempt to steer $x$ near zero in a short time. Equation (1) writes $\tau \dot{x}+x+k_{P} \operatorname{sgn} x(t-h)=0$ (resp. $\tau \ddot{x}+\dot{x}+k_{I} \operatorname{sgn} x(t-h)=0$ ) when $k_{I}=0\left(\right.$ resp. $\left.k_{P}=0\right)$. When $k_{P}=k_{I} \tau$ and $x(0)=-k_{I} y(0)$, one has $\dot{x}=-k_{I} \operatorname{sgn} x(t-h)$, giving saw-tooth evolution for $x$.

The motivation for considering such a control system comes from some automotive control problem, namely the fuel-air ratio regulation problem for spark ignition engine $[7,5]$. Another point of interest is the identification of the linear plant of the system on the basis of the limit cycle characteristics.

As explained in [3], sgn0 must be chosen single-valued, in order to have existence and uniqueness for the Cauchy problem. To eliminate the unstable equilibrium $x \equiv 0$, one chooses in the present paper:

$$
\operatorname{sgn} 0=1 .
$$

An important consequence of this choice (see Theorem 5) is that for any solution of (1) (by definition, any pair of absolutely continuous functions verifying (1) for almost every $t \geq 0$ ),

$$
\text { meas }\{t \geq 0: x(t)=0\}=0 .
$$

Let us give some definitions, useful to describe the periodic solutions properties.

Definition 1 (Slowly Oscillating (SO) functions ${ }^{1}$ ). A continuous function $x$ defined on $\left[t_{0},+\infty\right.$ [ is called slowly oscillating (with respect to $h$ ) if $x(t)=x\left(t^{\prime}\right)=0$ for $t, t^{\prime}>t_{0}$, $t \neq t^{\prime}$ implies $\left|t-t^{\prime}\right|>h$.

\footnotetext{
${ }^{1}$ See [14] for a survey on this notion in the context of periodic solutions of autonomous differential equations with delay.
}

$\mathrm{RR} \mathrm{n}^{\circ} 3422$ 
Definition 2 (2-Phase Periodic (2PP) functions [16]). A $T$-periodic $(T>0)$ continuous function $x$ defined on $\left[t_{0},+\infty\right)$ is called 2-phase periodic if there exists $t \geq t_{0}$ such that $\left.x\right|_{(t, t+T)}$ changes sign (strictly) exactly once.

Definition 3 (Symmetric Periodic functions). A $T$-periodic $(T>0)$ continuous function $x$ defined on $\left[t_{0},+\infty\right.$ is called symmetric if $x\left(t+\frac{T}{2}\right)=-x(t)$ for $t \geq t_{0}$.

We study system (1) under one of the following assumptions:

$$
\begin{gathered}
k_{I}=0, \quad k_{P}>0 \quad \text { (Proportional Control) } \\
k_{I}>0 \quad \text { (Proportional+Integral Control) }
\end{gathered}
$$

In a recent paper [3], we have shown that, under any of these hypotheses, the corresponding Cauchy problem admits a unique solution, and that there exists a SO periodic solution of (1). This solution is unique in the class of SO periodic solutions (up to time translation), asymptotically orbitally stable, symmetric and $2 \mathrm{PP}$. We have also shown how to control the characteristics of this limit cycle (period, amplitude) by an adequate choice of the control law parameters, and how to identify the linear plant by measuring the limit cycle characteristics. Also, were given therein a priori bounds on the solutions of the Cauchy problem associated with (1).

In the present paper, we present more precise informations on the dynamics.

In Section 1, we present the case where $\tau=0$. Here the periods of the different periodic solutions may be determined by elementary geometrical constructions. Their number is infinite countable, one of them only being SO.

In Section 2, the case where $\tau \neq 0$ is studied and proved to be qualitatively the same. We give, in the case $(\mathrm{P})$, an exhaustive description of the asymptotic behavior. More precisely, we show in Theorem 2 that the non-SO 2PP solutions are unstable, and that super-highfrequency solutions [17] (i.e. solutions having infinite number of zeros on any interval of $[0,+\infty)$ of length $h)$ do not exist. This permits to show that the proportional control law ensures that the mean-value of the solution goes asymptotically to zero (Corollary 3 ). The proof of Theorem 2 is postponed to Sections 4 and 5 .

In Section 3, one introduces perturbations of the nominal system (1): the parameters $h, \tau$ of the plant are now functions of time, and time-dependent errors $\zeta$ and $\xi$ are added respectively to the command and to the sign input (see system (5)). One shows that the Cauchy problem associated with this new system admits a unique solution, and provides asymptotic bounds. These estimates show the possibility to reject the influence of the perturbations (Theorem 6 and Corollary 7 ).

For a general overview on periodic solutions of autonomous delayed equations, we refer the reader to [6, Chapters XV and XVI].

INRIA 


\section{The particular case $\tau=0$}

When $\tau=0$ and (PI) holds, that is $k_{I}>0$, (1) writes

$$
x=-k_{P} \dot{y}-k_{I} y, \quad \dot{y}=\operatorname{sgn} x(t-h) .
$$

The curves $x(t)$ are piecewise affine, with slopes $\pm k_{I}$, and undergo jumps of magnitude $\pm 2 k_{P}$. Moreover, a change of mode at time $t$ implies a change of sign of $x$ at time $t-h$.

We construct $2 \mathrm{PP}$ symmetric solutions with period $T_{n}^{*}$ satisfying

$$
n T_{n}^{*} \leq h<(n+1) T_{n}^{*} .
$$

Here, $n=\left\lfloor\frac{h}{T_{*}^{*}}\right\rfloor$ : in other words, $n$ is the maximal number of periods included in an interval of length $h$. Geometric constructions shown in Figure 1 permit to determine the period $T_{0}^{*}$.
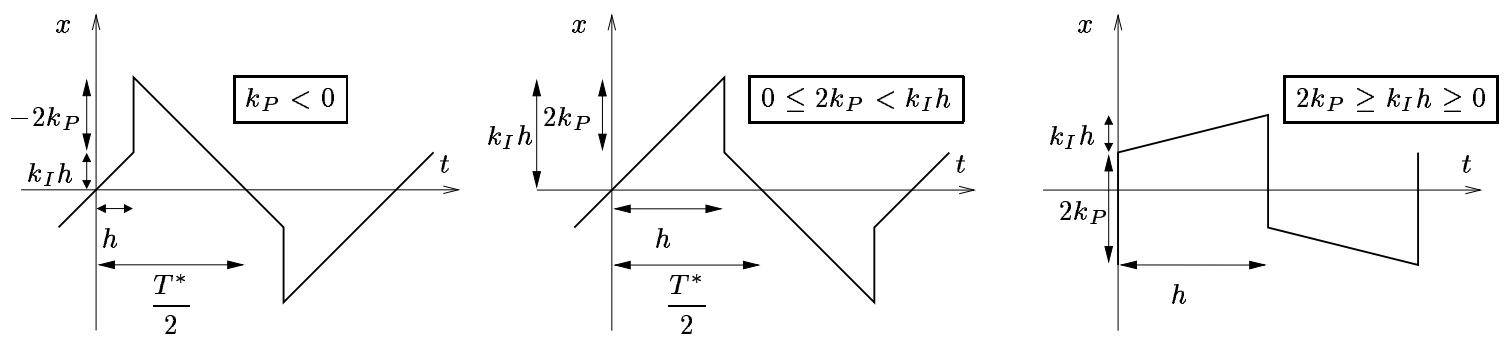

Figure 1: Computation of the period $T^{*}$ of the 2PP symmetric limit cycles when $\tau=0$. Case $T^{*} \geq 2 h$

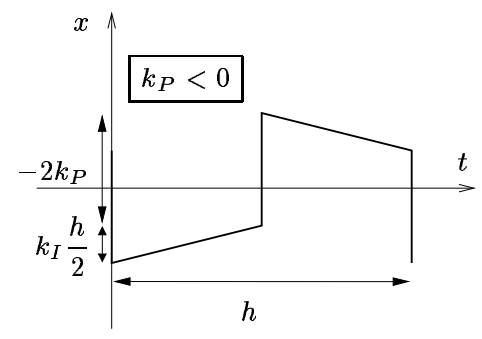

Figure 2: Computation of the period $T^{*}$ of the $2 \mathrm{PP}$ symmetric limit cycles when $\tau=0$. Case $h \leq T^{*}<2 h$

When $2 k_{P}<k_{I} h$ (resp. $\left.2 k_{P} \geq k_{I} h\right)$, one has

$$
\frac{T_{0}^{*}}{2}=h+\frac{k_{I} h-2 k_{P}}{k_{I}} \quad\left(\text { resp. } \frac{T_{0}^{*}}{2}=h\right) .
$$

$\mathrm{RR} \mathrm{n}^{\circ} 3422$ 
So one deduces the value of the period $T_{0}^{*}$ :

$$
T_{0}^{*}=\max \left\{2 h, 4\left(h-\frac{k_{P}}{k_{I}}\right)\right\} .
$$

The corresponding solution is SO if and only if $h>2 \frac{k_{P}}{k_{I}}$.

For the other $2 \mathrm{PP}$ symmetric solutions, the changes of mode are not consequence of the last change of sign of $x$, but of the last change situated before an entire number of halfperiods. When $0<h-n T_{n}^{*} \leq \frac{T_{n}^{*}}{2}$, then we obtain the corresponding period $T_{n}^{*}$ by replacing $h$ by $h-n T_{n}^{*}$ in (2), under the constraint that $h-n T_{n}^{*}>0$. We get

$$
T_{n}^{*}=\max \left\{\frac{1}{n+\frac{1}{2}} h, \frac{1}{n+\frac{1}{4}}\left(h-\frac{k_{P}}{k_{I}}\right)\right\}, T_{n}^{*}<\frac{h}{n}, n \in \mathbb{N},
$$

which corresponds to the portion of the curve given in Figure 3 for $\frac{k_{P}}{k_{I}}>-\frac{h}{4 n}$. There is no solution with $\frac{T_{n}^{*}}{2}<h-n T_{n}^{*}<T_{n}^{*}$.

When $h-n T_{n}^{*}=0$, then we get solutions of the type shown in Figure 2, leading to periods

$$
T_{n}^{*}=\frac{h}{n}, \frac{k_{P}}{k_{I}} \leq-\frac{h}{4 n}, n \in \mathbb{N}-\{0\},
$$

which correspond to the remainder of the curve given in Figure 3. The curve in this Figure is hence depicted by the following formula:

$$
\forall n \in \mathbb{N}, T_{n}^{*}=\min \left\{\frac{h}{n}, \max \left\{\frac{1}{n+\frac{1}{2}} h, \frac{1}{n+\frac{1}{4}}\left(h-\frac{k_{P}}{k_{I}}\right)\right\}\right\} .
$$

We obtain a countable number of branchs of solutions. For any value of $n \in \mathbb{N}, T_{n}^{*}$ is uniquely defined, and is a continuous nonincreasing function of $\frac{k_{P}}{k_{I}}$. The branch corresponding to $n=0$ is unbounded for $k_{P} / k_{I} \rightarrow-\infty$. The periods obtained for $n \neq 0$ are smaller than $2 h$, so they give rise to non-slowly oscillating periodic solutions.

One may also compute graphically the amplitude of the cycles. The result is given in Figure 4 . The curve is given analytically by

$$
\forall n \in \mathbb{N}, \quad\left\|x_{n}^{*}\right\|_{\infty}= \begin{cases}-k_{P}+\frac{1}{4 n} k_{I} h & \text { when } \frac{k_{P}}{k_{I}} \leq-\frac{h}{4 n}, \\ -\frac{2(2 n+1)}{4 n+1} k_{P}+\frac{1}{4 n+1} k_{I} h & \text { when }-\frac{h}{4 n} \leq \frac{k_{P}}{k_{I}} \leq 0, \\ \frac{4 n}{4 n+1} k_{P}+\frac{1}{4 n+1} k_{I} h & \text { when } 0 \leq \frac{k_{P}}{k_{I}} \leq \frac{h}{4\left(n+\frac{1}{2}\right)} \\ k_{P}+\frac{1}{2(2 n+1)} k_{I} h & \text { when } \frac{h}{4\left(n+\frac{1}{2}\right)} \leq \frac{k_{P}}{k_{I}}\end{cases}
$$

When $(\mathrm{P})$ holds, that is $k_{I}=0, k_{P}>0$, the same considerations show that the possible periods are $\frac{h}{n+\frac{1}{2}}, n \in \mathbb{N}$ : they are obtained in Figure 3 by taking the value $T_{n}^{*}$ corresponding to $\frac{k_{P}}{k_{I}} \rightarrow+\infty$. The amplitudes are obtained in the same manner. 


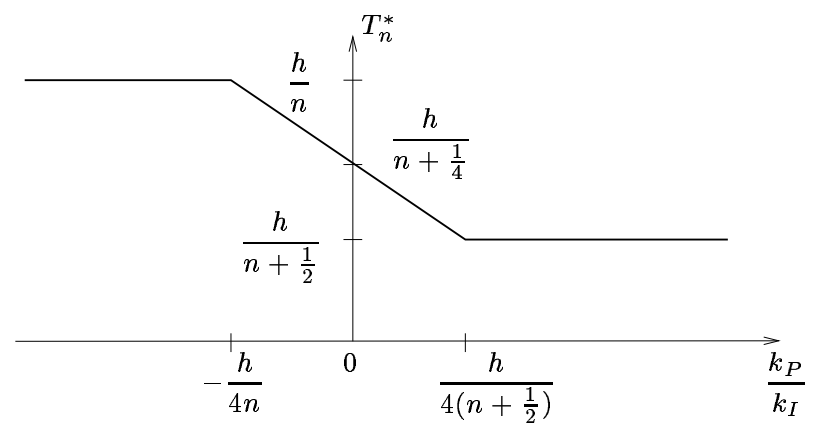

Figure 3: Periods of the $n$th branch of cycles when $\tau=0$ (the decreasing part is infinite on the left if $n=0$ )

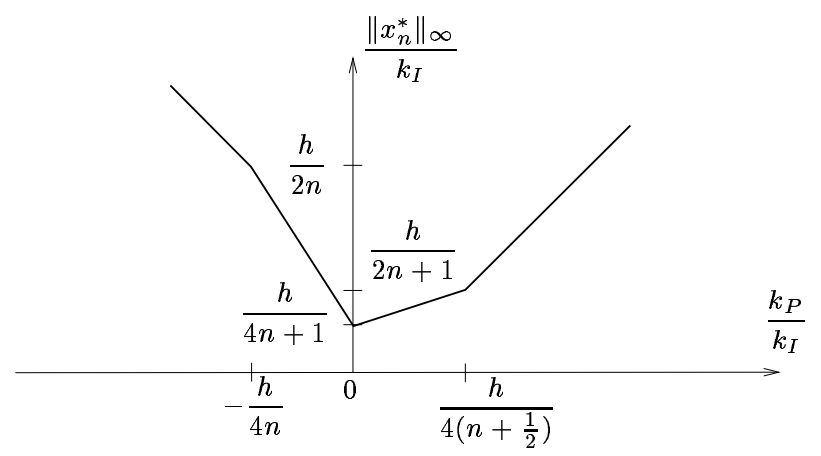

Figure 4: Amplitudes of the $n$th branch of cycles when $\tau=0$ (from left to right, the slopes are successively $-1,-1-\frac{1}{4 n+1}, 1-\frac{1}{4 n+1}, 1$ )

\section{The flow and its attractors in the general case $\tau \neq 0$}

In the sequel, we denote by a bar over a symbol the normalization by $\tau$ :

$$
\bar{h} \triangleq \frac{h}{\tau}, \bar{T} \triangleq \frac{T}{\tau} .
$$

Theorem 1 (Number and period of the 2PP solutions). If (P) or (PI) holds, then the periodic solutions $(x, y)$ of system (1) such that $x$ is $2 P P$, are symmetric and form (up to time-translation) an infinite countable set, denoted $\left\{\left(x_{n}^{*}, y_{n}^{*}\right)\right\}_{n \in \mathbb{N}}$. The solution with $n=0$ is the unique periodic solution such that $x$ is $S O$.

$\mathrm{RR} \mathrm{n}^{\circ} 3422$ 
The period $T_{n}^{*}$ of $\left(x_{n}^{*}, y_{n}^{*}\right)$ is defined uniquely by the following algebraic system:

$$
k_{I} \tau\left(\left(n+\frac{1}{4}\right) \bar{T}_{n}^{*}-\bar{h}\right)=\left(k_{I} \tau-k_{P}\right)\left(1-\frac{2}{1+e^{-\frac{T_{n}^{*}}{2}}} e^{-\left(n+\frac{1}{2}\right) \bar{T}_{n}^{*}+\bar{h}}\right), \quad \frac{\bar{h}}{n+\frac{1}{2}}<\bar{T}_{n}^{*} \leq \frac{\bar{h}}{n} .
$$

The solution $\left(x_{n}^{*}, y_{n}^{*}\right)$ is defined by the symmetry property and, for $0 \leq t<\frac{T_{n}^{*}}{2}$ :

$$
x_{n}^{*}(t)=k_{I} \tau\left(\frac{\bar{T}_{n}^{*}}{4}-\frac{t}{\tau}\right)+\left(k_{I} \tau-k_{P}\right)\left(1-\frac{2}{1+e^{-\frac{\bar{T}_{n}^{*}}{2}}} e^{-\frac{t}{\tau}}\right), y_{n}^{*}(t)=t-\frac{T_{n}^{*}}{4} .
$$

The situation when $\tau \neq 0$ is hence qualitatively the same as in Section 1; the curves, analogous to those shown in Figures 1 to 4, are smoother.

For any $n \in \mathbb{N}$, (3) defines a branch of solutions $\bar{T}_{n}^{*}$, which is continuous and decreasing as a function of $\frac{k_{P}}{k_{I}} \in(-\infty,+\infty]$. When $\frac{k_{P}}{k_{I}}=\tau$, one has $T_{n}^{*}=\frac{h}{n+\frac{1}{4}}$, as in the case $\tau=0$ (see Figure 3).

Remark that the value of the period may be deduced from Tsypkin's results [20].

Sketch of Proof of Theorem 1. The proof is conducted as follows (see details of the computation of the slowly oscillating solution in [3]). Let $(x, y)$ be a periodic solution of (1) with $x$ $2 \mathrm{PP}$. One deduces from the periodicity of $y$ that $\operatorname{sgn} x$ is symmetric. Suppose that $\left(x_{n}^{*}, y_{n}^{*}\right)$ is such a solution, with $x_{n}^{*}(t) \geq 0$ for $t \in\left[-h, \frac{T_{n}^{*}}{2}-h\right], x_{n}^{*}(t) \leq 0$ for $t \in\left[\frac{T_{n}^{*}}{2}-h, T_{n}^{*}-h\right]$, and $n T_{n}^{*} \leq h<(n+1) T_{n}^{*}$. Solving the equations piecewise and expressing the continuity and periodicity conditions for $x_{n}^{*}$ and $y_{n}^{*}$ leads to the symmetry of $x$ and $y$, and some necessary conditions on $T_{n}^{*}$. Expressing compatibility conditions on $\operatorname{sgn} x_{n}^{*}$, we get (3), which is also shown to be sufficient. This method also provides explicit form of the corresponding periodic solutions.

As said in the Introduction, it is shown in [3] that one may choose $k_{P}, k_{I}$ in order that the slowly oscillating limit cycle (corresponding to $n=0$ ) has any prescribed amplitude and period greater than the value of $T_{0}^{*}$ in the case $(\mathrm{P})$. In particular, the negative values of $k_{P}$ are necessary to realize large values of the period. The slowly oscillating limit cycle is the only that one observes numerically. We proved in [3] that it is asymptotically orbitally stable. We now go on studying the asymptotics.

We define the set of points where the solution $x$ of (1) vanishes and changes sign, and the cardinal of the number of zeros with changes of sign:

$$
\begin{gathered}
Z \triangleq\left\{t \geq 0: x(t)=0 \text { and } \forall \varepsilon>0, \exists t^{\prime} \in[t-\varepsilon, t), t^{\prime \prime} \in(t, t+\varepsilon], x\left(t^{\prime}\right) x\left(t^{\prime \prime}\right)<0\right\}, \\
V(t)=\left\{\begin{array}{lc}
\operatorname{card} Z \cap\left[t^{\prime}-h, t^{\prime}\right) & \text { when }[0, t] \cap Z \neq \emptyset, t^{\prime}=\sup [0, t] \cap Z \geq h \\
+\infty & \text { and } Z \cap\left[t^{\prime}-h, t^{\prime}\right) \text { is finite }
\end{array}\right.
\end{gathered}
$$


One has obviously for any $t \geq 0: V(t) \in \mathbb{N} \cup\{+\infty\}$. In $[9,17]$, similar constructions are made. The set $Z$ is unbounded [9,3], and we shall see in the proof of Theorem 2 (Lemma 10) that it is closed.

Remark that $V(t)=0$ for $t$ large enough if and only if $x$ is slowly oscillating on an interval of the form $\left[t_{0},+\infty\right)$.

Theorem 2 (Behavior of the solutions in the case $(\mathrm{P})$ ). Suppose $(\mathrm{P})$ holds. Then, for any solution of the Cauchy problem associated with (1), V(t) takes on nonincreasing even values, finite after a finite time.

Denote $2 n=\lim _{t \rightarrow+\infty} V(t)$, and let $\underline{t}$ be the smallest time $t$ for which $V(t)=2 n$ (so that $V(t)=2 n$ for $t \geq \underline{t})$. Then, there exists $\varphi \geq 0$ such that

$$
t \geq \underline{t} \Rightarrow x(t)=x_{n}^{*}(t+\varphi) .
$$

The SO cycle is asymptotically orbitally stable, the non-SO cycles are unstable.

In particular, Theorem 2 implies that, in the case $(\mathrm{P})$, the only periodic solutions are the ones exhibited in Theorem 1.

The fact that $V(t)$ is finite after a certain time (disappearance of super-high-frequency oscillations [17]) is obtained without condition on the delay. This improves the result in [17]. In $[1,2]$, this result is generalized. Also, some results may be generalized to higher-order systems $[18,10]$.

The case $\tau=0$ appears as degenerated, as the slowest periodic solution is not SO, since its period is $2 h$ (as $\left.k_{I}=0\right)$.

Corollary 3. If (P) holds, then, for any trajectory of (1),

$$
\begin{gathered}
\lim _{t^{\prime} \rightarrow+\infty} \sup \left\{t-t^{\prime}: t \geq t^{\prime}, x \geq 0(\text { resp. } \leq 0) \text { on }\left[t^{\prime}, t\right]\right\} \leq \frac{T_{0}^{*}}{2}, \\
\lim _{t \rightarrow+\infty} \frac{1}{t} \int_{0}^{t} x(s) d s=\lim _{t \rightarrow+\infty} \frac{1}{t} \int_{0}^{t} \operatorname{sgn} x(s) d s=0 .
\end{gathered}
$$

Corollary 3 shows that in the case $(\mathrm{P})$, the mean value of $x$ is controlled to zero. Remark that, more generally, when $(\mathrm{P})$ holds, the asymptotic properties of the solutions need only to be checked for the periodic ones. As an example,

$$
\limsup _{t \rightarrow+\infty}|x(t)| \leq\left\|x_{0}^{*}\right\|_{\infty}=k_{P}\left(1-e^{-\bar{h}}\right) .
$$

The proof of Theorem 2 is postponed to Sections 4 and 5. In Section 4, we demonstrate the results related to solutions with finite number of zeros, with techniques analogous to what is done in [8], whereas Section 5 is devoted to the solutions with infinite number of zeros, using the techniques of [17] in an adapted version. 


\section{Estimates of the solutions of the Cauchy problem ro- bust wrt perturbations of the parameters}

We now consider a perturbed version of (1), namely the system:

$$
\left\{\begin{array}{l}
\dot{x}(t)=-\frac{1}{\tau(t)}\left(k_{I} y(t)+k_{P} \operatorname{sgn}[x(t-h(t))+\xi(t-h(t))]+x(t)+\zeta(t)\right) \\
\dot{y}(t)=\operatorname{sgn}[x(t-h(t))+\xi(t-h(t))]
\end{array}\right.
$$

Theorem 4 (Existence and uniqueness for solutions of (5)). Suppose that $(\mathrm{P})$ or $(\mathrm{PI})$ holds, that $\mathrm{Id}-h$ is nondecreasing with $h\left(t^{+}\right)>0$ for any $t \geq 0$, that $\tau$ is nonnegative and such that $\frac{1}{\tau} \in L_{\mathrm{loc}}^{1}((0,+\infty))$, and that $\zeta, \xi \in L_{\mathrm{loc}}^{1}((0,+\infty))$. Then, for any $\left(x_{0}, x_{00}, y_{0}\right) \in$ $L^{1}((-h(0+), 0)) \times \mathbb{R} \times \mathbb{R}$, there exists a unique pair $(x, y) \in L_{\mathrm{loc}}^{1}((-h(0+),+\infty)) \times W_{\mathrm{loc}}^{1, \infty}([0,+\infty))$, such that $x \in W_{\mathrm{loc}}^{1, \infty}([0,+\infty)),\left.x\right|_{(-h(0+), 0)}=x_{0}, x(0)=x_{00}, y(0)=y_{0}$, and $(x, y)$ verifies equation (5) for almost every $t \in \mathbb{R}^{+}$.

Proof. The fact that Id $-h$ is nondecreasing implies that $h$ has left- and right-limits on any point $t$, with $h\left(t^{+}\right) \leq h(t) \leq h\left(t^{-}\right)$. This property and the fact that $h\left(t^{+}\right)>0$ implies that we cannot have $t_{n} \rightarrow t$ with $h\left(t_{n}^{+}\right) \rightarrow 0$. Therefore, $h\left(t^{+}\right)$, and then $h(t)$, is bounded from below on any compact of $[0,+\infty)$. Existence and uniqueness uses the fact that $h$ is locally bounded from below: the integration is performed on intervals of length $h(t)$, using the local integrability of $\frac{1}{\tau}$.

Theorem 5 (Zeros of the solutions of (5)). Suppose that the hypotheses of Theorem 4 are fulfilled and that $\tau$ is finite a.e. on $\mathbb{R}^{+}$.

- If meas $\left\{t \geq 0:|\zeta(t)|=k_{P}\right\}=0$ in the case $(\mathrm{P})$, or if $\zeta$ is differentiable a.e. with meas $\left\{t \geq 0:\left|\frac{d}{d t} \zeta(t)\right|=k_{I}\right\}=0$ in the case (PI), then, for all $\left(x_{0}, x_{00}, y_{0}\right) \in$ $L^{1}((-h(0+), 0)) \times \mathbb{R} \times \mathbb{R}$,

$$
\text { meas }\{t \geq 0: x(t)=0\}=0 .
$$

- If $\xi$ is absolutely continuous, if meas $\left\{t \geq 0:|\tau(t) \dot{\xi}(t)+\xi(t)+\zeta(t)|=k_{P}\right\}=0$ in the case $(\mathrm{P})$, or if $\tau \dot{\xi}+\xi+\zeta$ is differentiable a.e. with meas $\left\{t \geq 0:\left|\frac{d}{d t}(\tau \dot{\xi}+\xi+\zeta)\right|=\right.$ $\left.k_{I}\right\}=0$ in the case $(\mathrm{PI})$, then, for all $\left(x_{0}, x_{00}, y_{0}\right) \in L^{1}((-h(0+), 0)) \times \mathbb{R} \times \mathbb{R}$,

$$
\text { meas }\{t \geq 0: x(t)+\xi(t)=0\}=0 .
$$

When (7) is fulfilled, then the solutions of (5) do not depend upon the definition of sgn0 (the same result would be true if $\operatorname{sgn} 0$ was defined as a measurable function of $t \in[0,+\infty)$ with values 1 or -1 a.e.). Conversely, one may show that if on a certain time-interval, $\tau \dot{\xi}+\xi+\zeta=k_{P}$ a.e. in the case (P), or $\frac{d}{d t}(\tau \dot{\xi}+\xi+\zeta)=k_{I}$ a.e. in the case (PI), then, some trajectories depend upon the choice of $\operatorname{sgn} 0$. 
Proof of Theorem 5. To prove property (6), suppose first that $k_{I}=0$. Denote $N \subset \mathbb{R}^{+}$a set of measure zero such that outside $N, x$ is differentiable, (5) is fulfilled, $\tau \neq+\infty$ and $|\zeta| \neq k_{P}$. For any $\varepsilon>0$, there exists an open set $U$ such that $N \subset U$ and meas $U<\varepsilon$. Denote $S \triangleq\{t \geq 0: x(t)=0\}$ and $N^{\mathrm{c}} \triangleq\{t \geq 0: t \notin N\}, U^{\mathrm{c}} \triangleq\{t \geq 0: t \notin U\}$. It is clear that $S \cap N^{c}$ has no accumulation point, otherwise on such a point $t$, one would have $x(t)=0, \dot{x}(t)=0$, so $k_{P} \dot{y}(t)+\zeta(t)=0$, together with $|\dot{y}(t)|=1$. As $S \cap U^{\mathrm{c}} \subset S \cap N^{\mathrm{c}}$, the set $S \cap U^{\mathrm{c}}$ has no point of accumulation. Being closed (by the continuity of $x$ ), it is then finite or countable. Hence, meas $\left(S \cap U^{\mathrm{c}}\right)=0$, so meas $S=$ meas $(S \cap U)<\varepsilon$ for any $\varepsilon>0$.

Suppose now $k_{I} \neq 0$. We define the set $N$ with the same properties as before, with the condition $|\zeta| \neq k_{P}$ replaced by: $\zeta$ differentiable and $|\dot{\zeta}| \neq k_{I}$. The set $U$ is then defined as before. First, the set $\left\{t \in N^{\mathrm{c}}: k_{P} \dot{y}(t)+k_{I} y(t)+\zeta(t)=0\right\}$ has no accumulation point (because on such a point, we would have $\left.k_{I} \dot{y}(t)+\dot{\zeta}(t)=0\right)$. As the later set contains the set of accumulation points of $S \cap N^{\mathrm{c}}$, and hence of the closed set $S \cap U^{\mathrm{c}}$, there exists an open set $V$ containing the accumulation points of $S \cap U^{\mathrm{c}}$ and such that meas $V<\varepsilon$. Denote $V^{\mathrm{c}} \triangleq\{t \geq 0: t \notin V\}$. Because the set $S \cap U^{\mathrm{c}} \cap V^{\mathrm{c}}$ has no accumulation point, we have meas $\left(S \cap U^{\mathrm{c}} \cap V^{\mathrm{c}}\right)=0$, so meas $S \leq$ meas $(S \cap U)+$ meas $(S \cap V) \leq 2 \varepsilon$ for any $\varepsilon>0$.

The proof of property $(7)$ is conducted similarly. The continuity of $\xi$ is necessary to claim that the set $\{t \geq 0: x(t)+\xi(t)=0\}$ is closed.

Since we consider only the behavior at infinity of the solutions, we consider the following seminorms on $L^{\infty}((0,+\infty))$ (the subscript $a$ is for asymptotic and sup, inf mean supess, inf ess):

$$
\|x\|_{a} \triangleq \limsup _{t \rightarrow+\infty}|x(t)|, \quad|x|_{a} \triangleq \frac{1}{2}\left(\limsup _{t \rightarrow+\infty} x(t)-\liminf _{t \rightarrow+\infty} x(t)\right)
$$

In the following results, we use the notations $z^{+} \triangleq \max \{z, 0\}, z^{-} \triangleq \max \{-z, 0\}$.

Theorem 6 (Estimates for solutions of (5)). Suppose that the hypotheses of Theorem 4 are fulfilled and that $\zeta, \xi, h, \tau \in L^{\infty}((0,+\infty))$, with

$$
|\zeta|_{a} \leq \delta_{\zeta},|\xi|_{a} \leq \delta_{\xi},\|\zeta\|_{a} \leq \Delta_{\zeta},\|\xi\|_{a} \leq \Delta_{\xi},
$$

for some constants $\delta_{\zeta}, \delta_{\xi}, \Delta_{\zeta}, \Delta_{\xi}>0$. Let $\left(x_{0}, x_{00}, y_{0}\right) \in L^{1}((-h(0+), 0)) \times \mathbb{R} \times \mathbb{R}$.

- In the case $(\mathrm{P})$, then

$$
\begin{gathered}
|x|_{a} \leq k_{P}+\delta_{\zeta}-e^{-\frac{\|h\| a}{\lim \text { inf } \tau}} \min \left\{2 k_{P},\left(k_{P}+\delta_{\zeta}-\delta_{\xi}\right)^{+},\left(\frac{3}{2} k_{P}+\delta_{\zeta}-\frac{\Delta_{\zeta}}{2}-\frac{\Delta_{\xi}}{2}\right)^{+}\right\}, \\
\|x\|_{a} \leq k_{P}+\Delta_{\zeta}-e^{-\frac{\|h\| a}{\liminf \tau}} \min \left\{2 k_{P},\left(k_{P}+\Delta_{\zeta}-\Delta_{\xi}\right)^{+}\right\}
\end{gathered}
$$

- In the case (PI), then

$$
|x|_{a} \leq \delta_{\xi}+2 \Phi\left(\max \left\{\hat{T}, \tilde{T}, 2\|h\|_{a}\right\}\right), \quad\|x\|_{a} \leq \Delta_{\xi}+2 \Phi\left(\max \left\{\hat{T}, \tilde{T}, 2\|h\|_{a}\right\}\right)
$$


where

$$
\begin{aligned}
\Phi(T) & \triangleq-k_{I}\left(\frac{T}{4}-\|h\|_{a}\right)+K_{2}+K_{3}^{+} e^{-\frac{T}{2}-\|h\|_{a}}-K_{3}^{-} e^{-\frac{\frac{T}{2}-\|h\|_{a}}{\lim \lim \sup \tau}}, \\
K_{1} & \triangleq \frac{1}{\liminf \tau}\left(\left\|k_{I} \tau-k_{P}\right\|_{a}+\left|k_{P}\right|+2 \delta_{\zeta}\right), \\
K_{2} & \triangleq \frac{1}{2}\left(\left\|k_{I} \tau-k_{P}\right\|_{a}+\lim \sup \left(k_{I} \tau-k_{P}\right)\right)+\delta_{\zeta}, \\
K_{3} & \triangleq \frac{1}{2}\left(\left\|k_{I} \tau-k_{P}\right\|_{a}-\lim \sup \left(k_{I} \tau-k_{P}\right)\right)-e^{-\frac{\|h\| a}{\lim \operatorname{lin} \tau}}\left(\left\|k_{I} \tau-k_{P}\right\|_{a}+\delta_{\zeta}\right),
\end{aligned}
$$

and $\hat{T}, \tilde{T}$ are defined by

$$
\begin{aligned}
& \Phi(\hat{T})=K_{1} \frac{\hat{T}}{4}, \quad \hat{T}>0, \\
& \frac{\tilde{T}}{2} \triangleq\|h\|_{a}+\liminf \tau \log \left(\frac{2 K_{3}^{-}}{k_{I} \lim \inf \tau}\right) .
\end{aligned}
$$

Estimates for $y$ may be deduced from estimates on $x+k_{I} y$ (see the proof).

The results of Theorem 6 are to be compared with the open-loop system $\left(k_{P}=0, k_{I}=0\right)$, for which the worst-case estimate are $|x|_{a}=|\zeta|_{a},\|x\|_{a}=\|\zeta\|_{a}$. The Proportional control permits in certain cases to reject the perturbation. However, it is not possible to render $\|x\|_{a}$ small when $|\zeta|_{a}=0$ only. This can be achieved by the Proportional-Integral control, as shown in the following result.

Corollary 7 (Rejection of the perturbations). Let us use the same notations and assumptions as in Theorem 6 , and suppose that

$$
\|\tau\|_{a}>4|\tau|_{a} .
$$

If the control parameters are chosen in such a way that

$$
k_{P}=k_{I}\left(\|\tau\|_{a}-|\tau|_{a}\right), \quad k_{I} \geq \frac{2 \delta_{\zeta}}{\|\tau\|_{a}-4|\tau|_{a}},
$$

then,

$$
|x|_{a} \leq \delta_{\xi}+2 k_{I}\|h\|_{a},\|x\|_{a} \leq \Delta_{\xi}+2 k_{I}\|h\|_{a} .
$$

Corollary 7 demonstrates that the perturbation $\zeta$ may be rejected when the measurement noise $\xi$ is "small" wrt the model error $\zeta$, the delay $h$ is "small" wrt $\tau$, and $\tau$ "does not vary too much". In particular, the integral term permits to reject the constant perturbations $\zeta$.

In order to prove Theorem 6, we gather some estimates in the following technical result, which gives indeed more informations than what is needed.

INRIA 
Proposition 8. Under the hypotheses of Theorem 6 , for all $\left(x_{0}, x_{00}, y_{0}\right) \in L^{1}((-h(0+), 0)) \times$ $\mathbb{R} \times \mathbb{R}$, the following estimates hold.

- In the case $(\mathrm{P})$, we have

$\lim \sup x \leq k_{P}-\liminf \zeta-e^{-\frac{\|h\| a}{\lim \inf \tau}} \min \left\{2 k_{P},\left(k_{P}-\liminf \zeta+\liminf \xi\right)^{+}\right\}$.

Moreover, if

$$
k_{P}>\lim \sup \xi-\liminf \zeta,
$$

then

$$
\begin{aligned}
& \limsup _{t^{\prime} \rightarrow+\infty} \sup \left\{t-t^{\prime}: t \geq t^{\prime}, x+\xi>0 \text { a.e. on }\left(t^{\prime}, t\right)\right\} \\
& \quad \leq(\lim \sup \tau)\left(\log \left(\frac{k_{P}\left(2 e^{\frac{\|h\| a}{\lim \operatorname{nin} \tau}}-1\right)+\min \left\{\lim \inf \zeta-\lim \inf \xi, k_{P}\right\}}{k_{P}+\lim \inf \zeta-\lim \sup \xi}\right)\right)^{+} .
\end{aligned}
$$

- In the case (PI), defining

$$
\begin{aligned}
K_{1} & \triangleq \frac{1}{\liminf \tau}\left(\left\|k_{I} \tau-k_{P}\right\|_{a}+\left|k_{P}\right|+2|\zeta|_{a}\right), \\
K_{2} & \triangleq \frac{1}{2}\left(\left\|k_{I} \tau-k_{P}\right\|_{a}+\lim \sup \left(k_{I} \tau-k_{P}\right)\right)+|\zeta|_{a}, \\
K_{3} & \triangleq \frac{1}{2}\left(\left\|k_{I} \tau-k_{P}\right\|_{a}-\lim \sup \left(k_{I} \tau-k_{P}\right)\right)-e^{-\frac{\|h\|_{a}}{\lim \operatorname{in} \tau}}\left(\left\|k_{I} \tau-k_{P}\right\|_{a}+|\zeta|_{a}\right), \\
\Phi(T) & \triangleq-k_{I}\left(\frac{T}{4}-\|h\|_{a}\right)+K_{2}+K_{3}^{+} e^{-\frac{T}{2}-\|h\|_{a}}-K_{3}^{-} e^{-\frac{T}{2}-\|h\|_{a}},
\end{aligned}
$$

we have:

$$
\begin{gathered}
\lim \sup x \leq-\lim \inf \xi+2 \Phi\left(\max \left\{\hat{T}, \tilde{T}, 2\|h\|_{a}\right\}\right) \\
\limsup _{t^{\prime} \rightarrow+\infty} \sup \left\{t-t^{\prime}: t \geq t^{\prime}, x+\xi>0 \text { a.e. on }\left(t^{\prime}, t\right)\right\} \leq \frac{T^{+}}{2},
\end{gathered}
$$

where $\hat{T}, \tilde{T}, T^{+}$are defined by

$$
\begin{aligned}
\Phi(\hat{T}) & =K_{1} \frac{\hat{T}}{4}, \quad \hat{T}>0, \\
\frac{\tilde{T}}{2} & \triangleq\|h\|_{a}+\liminf \tau \log \left(\frac{2 K_{3}^{-}}{k_{I} \lim \inf \tau}\right), \\
\Phi\left(T^{+}\right) & =-|\xi|_{a}, \quad T^{+}>0 .
\end{aligned}
$$

$\mathrm{RR} \mathrm{n}^{\circ} 3422$ 
Proof of Theorem 6 . Results for liminf $x$ similar to (8) and (11) are obtained by changing $x, \zeta, \xi$ into their opposite; one hence gets estimates for $\|x\|_{a}$ and $|x|_{a}$. At last, one verifies that the obtained upper bounds are nondecreasing functions of $|\zeta|_{a},|\xi|_{a},\|\zeta\|_{a},\|\xi\|_{a}$, which permits to prove Theorem 6. This last step is clear for the case (P). For the case (PI), one uses the fact that

$$
2 \Phi\left(\max \left\{\hat{T}, \tilde{T}, 2\|h\|_{a}\right\}\right)=\sup _{T \geq 2\|h\|_{a}} \min \left\{K_{1} \frac{T}{2}, 2 \Phi(T)\right\},
$$

where $K_{1}$ and $\Phi(T)$ are nondecreasing functions of $|\zeta|_{a}$ when $T \geq 2\|h\|_{a}$.

The form under which are presented the results is close to the one used for the period in the unperturbed case (see formula (3) above). Remark that in the case (P), the estimates are optimal for the unperturbed system: they yield lim $\sup x \leq\left\|x_{0}^{*}\right\|_{\infty}$ and $\lim \sup _{t^{\prime} \rightarrow+\infty} \sup \{t-$ $t^{\prime}: t \geq t^{\prime}, x>0$ a.e. on $\left.\left(t^{\prime}, t\right)\right\} \leq \frac{T_{0}^{*}}{2}$, where $x_{0}^{*}$ is the slowly oscillating periodic solution and $T_{0}^{*}$ its period (see Corollary 3 ). In the case (PI), even in the unperturbed case, the estimates are less accurate, as $T^{+}$defined by (12) is greater than $T_{0}^{*}$. Indeed, computations are difficult, due to the fact that there exist slowly oscillating non periodic solutions (which tend asymptotically to $x_{0}^{*}$ ) [3], contrary to the case (P), where the convergence is effective after the first zero.

Proof of Proposition 8.

- We begin with the case (PI). We have

$$
\tau(t)\left(\dot{x}+k_{I} \dot{y}\right)+\left(x+k_{I} y\right)=\left(k_{I} \tau-k_{P}\right) \dot{y}-\zeta .
$$

Hence, using the fact that $\frac{1}{\tau} \notin L^{1}((0,+\infty))$, we get:

$$
\begin{array}{r}
-\left\|k_{I} \tau-k_{P}\right\|_{a}-\limsup \zeta \leq \liminf \left(x+k_{I} y\right) \\
\quad \leq \limsup \left(x+k_{I} y\right) \leq\left\|k_{I} \tau-k_{P}\right\|_{a}-\liminf \zeta, \\
\limsup \dot{x} \leq\left\|\frac{1}{\tau}\right\|_{a}\left(\left\|k_{I} \tau-k_{P}\right\|_{a}+\left|k_{P}\right|+2|\zeta|_{a}\right)=K_{1} .
\end{array}
$$

Define $X \triangleq x+\xi$. Firstly, there is no unbounded time-interval on which e.g. $X>0$ almost everywhere. Indeed, we would have $\dot{y}=1$ a.e. on an unbounded interval, so $x(t) \rightarrow-\infty$ when $t \rightarrow+\infty$, due to the boundedness of $\zeta$. The boundedness of $\xi$ then implies that $X(t) \rightarrow-\infty$, which contradicts the hypothesis.

Secondly, for any $\varepsilon>0$, there is a $t_{0}>0$ such that the lim sup which will be involved in the sequel are approached up to $\varepsilon$ for $t \geq t_{0}$. As we are interested in the asymptotic behavior only, we shall omit in the following the $\varepsilon$ 's, for sake of simplicity. 
Let $t^{\prime}, t$ be such that $\left(t_{0} \leq\right) t^{\prime}<t$ and $X>0$ almost everywhere on $\left(t^{\prime}, t\right)$. We may indeed suppose without loss of generality that $t^{\prime}=\inf \{s: X>0$ a.e. on $(s, t)\}$. This implies, due to the continuity of $x$, that

$$
x\left(t^{\prime}\right) \leq-\liminf \xi, x(t) \geq-\lim \sup \xi .
$$

Let us define

$$
t^{\prime \prime} \triangleq \sup \left\{s \in[0, t]: s-h(s) \leq t^{\prime}\right\} .
$$

One has $t^{\prime}<t^{\prime \prime} \leq t$ and $t^{\prime \prime}-h\left(t^{\prime \prime-}\right) \leq t^{\prime}$. Then,

$$
y(t)-y\left(t^{\prime}\right)=\int_{t^{\prime}}^{t^{\prime \prime}} \operatorname{sgn} X(s-h(s)) d s+t-t^{\prime \prime} \geq t-t^{\prime \prime}-\|h\|_{a},
$$

using the fact that $X(s-h(s))>0$ a.e. on $\left(t^{\prime \prime}, t\right)$. From (13), we deduce:

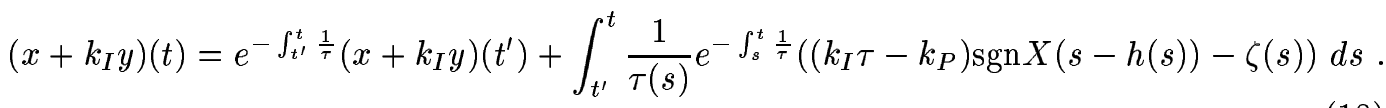

From inequalities (14) and (17), we obtain

$$
\begin{aligned}
& e^{-\int_{t^{\prime}}^{t} \frac{1}{\tau}}\left(x+k_{I} y\right)\left(t^{\prime}\right)-k_{I} y(t)-x\left(t^{\prime}\right) \\
& \quad=k_{I}\left(y\left(t^{\prime}\right)-y(t)\right)-\left(x\left(t^{\prime}\right)+k_{I} y\left(t^{\prime}\right)\right)\left(1-e^{-\int_{t^{\prime}}^{t} \frac{1}{\tau}}\right) \\
& \quad \leq k_{I}\left(\|h\|_{a}-\left(t-t^{\prime \prime}\right)\right)+\left(\left\|k_{I} \tau-k_{P}\right\|_{a}+\lim \sup \zeta\right)\left(1-e^{-\int_{t^{\prime}}^{t^{\prime \prime}} \frac{1}{\tau}} e^{-\int_{t^{\prime \prime}}^{t} \frac{1}{\tau}}\right)
\end{aligned}
$$

Also,

$$
\begin{aligned}
\int_{t^{\prime}}^{t} & \frac{1}{\tau(s)} e^{-\int_{s}^{t} \frac{1}{\tau}}\left(\left(k_{I} \tau-k_{P}\right) \operatorname{sgn} X(s-h(s))-\zeta(s)\right) d s \\
\leq & -(\liminf \zeta)\left(1-e^{-\int_{t^{\prime}}^{t} \frac{1}{\tau}}\right)+\lim \sup \left(k_{I} \tau-k_{P}\right)\left(1-e^{-\int_{t^{\prime \prime}}^{t} \frac{1}{\tau}}\right) \\
& +\left\|k_{I} \tau-k_{P}\right\|_{a}\left(e^{-\int_{t^{\prime \prime}}^{t} \frac{1}{\tau}}-e^{-\int_{t^{\prime}}^{t} \frac{1}{\tau}}\right) \\
= & \left(-\liminf \zeta+\lim \sup \left(k_{I} \tau-k_{P}\right)\right) \\
& +\left(\left\|k_{I} \tau-k_{P}\right\|_{a}-\lim \sup \left(k_{I} \tau-k_{P}\right)-\left(\left\|k_{I} \tau-k_{P}\right\|_{a}-\liminf \zeta\right) e^{-\int_{t^{\prime}}^{t^{\prime \prime}} \frac{1}{\tau}}\right) e^{-\int_{t^{\prime \prime}}^{t} \frac{1}{\tau}}
\end{aligned}
$$

$\mathrm{RR} \mathrm{n}^{\circ} 3422$ 
Adding the two inequalities, we deduce that:

$$
\begin{aligned}
& x(t)-x\left(t^{\prime}\right) \\
& \leq k_{I}\left(\|h\|_{a}-\left(t-t^{\prime \prime}\right)\right)+\left(\left\|k_{I} \tau-k_{P}\right\|_{a}+\lim \sup \left(k_{I} \tau-k_{P}\right)+2|\zeta|_{a}\right)
\end{aligned}
$$

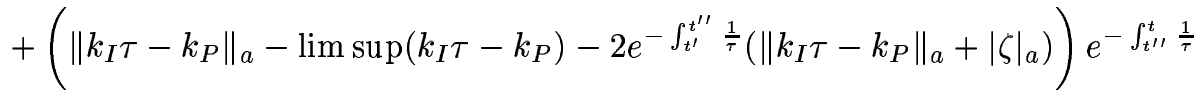

$$
\begin{aligned}
& =k_{I}\left(\|h\|_{a}-\left(t-t^{\prime \prime}\right)\right)+2 K_{2}+2 K_{3} e^{-\int_{t^{\prime \prime}}^{t} \frac{1}{\tau}} \\
& \leq k_{I}\left(\|h\|_{a}-\left(t-t^{\prime \prime}\right)\right)+2 K_{2}+2 K_{3}^{+} e^{-\frac{t-t^{\prime \prime}}{\lim \sup \tau}}-2 K_{3}^{-} e^{-\frac{t-t^{\prime \prime}}{\lim \inf \tau}} \\
& =2 \Phi\left(2\left(t-t^{\prime \prime}+\|h\|_{a}\right)\right),
\end{aligned}
$$

where $K_{2}, K_{3}, \Phi$ are defined in the statement of the proposition. Now, (16) implies that $x(t)-x\left(t^{\prime}\right) \geq-2|\xi|_{a}$, so $t-t^{\prime} \leq t-t^{\prime \prime}+\|h\|_{a} \leq \frac{T^{+}}{2}$, with $T^{+}$defined by formula (12).

Using (15), we get $x(t)-x\left(t^{\prime}\right) \leq K_{1}\left(t-t^{\prime}\right) \leq K_{1}\left(t-t^{\prime \prime}+\|h\|_{a}\right)$. Hence,

$$
x(t)-x\left(t^{\prime}\right) \leq \sup _{T \geq 2\|h\|_{a}} \min \left\{K_{1} \frac{T}{2}, 2 \Phi(T)\right\} .
$$

Using the fact that $\Phi$ decreases on $[\tilde{T},+\infty)$, we obtain

$$
\sup _{T \geq 2\|h\|_{a}} \min \left\{K_{1} \frac{T}{2}, 2 \Phi(T)\right\}=2 \Phi\left(\max \left\{\hat{T}, \tilde{T}, 2\|h\|_{a}\right\}\right),
$$

and the statement of the proposition follows from (16).

- Let us now consider the case $(\mathrm{P})$. With the same techniques as before, we estimate (18) and get:

$$
x(t) \leq x\left(t^{\prime}\right) e^{-\int_{t^{\prime}}^{t} \frac{1}{\tau}}-\left(\liminf \zeta+k_{P}\right)\left(1-e^{-\int_{t^{\prime}}^{t} \frac{1}{\tau}}\right)+2 k_{P}\left(e^{-\int_{t^{\prime \prime}}^{t} \frac{1}{\tau}}-e^{-\int_{t^{\prime}}^{t} \frac{1}{\tau}}\right) .
$$

From this, we deduce

$$
\begin{aligned}
& \lim \inf \zeta+k_{P}-\lim \sup \xi \\
& \quad \leq \liminf \zeta+k_{P}+x(t) \\
& \quad \leq\left(x\left(t^{\prime}\right)+\liminf \zeta+k_{P}+2 k_{P}\left(e^{\int_{t^{\prime}}^{t^{\prime \prime}} \frac{1}{\tau}}-1\right)\right) e^{-\int_{t^{\prime}}^{t} \frac{1}{\tau}} \\
& \quad \leq\left(\min \left\{-\lim \inf \xi, k_{P}-\lim \inf \zeta\right\}+\liminf \zeta+k_{P}\left(2 e^{\int_{t^{\prime}}^{t^{\prime \prime}} \frac{1}{\tau}}-1\right)\right) e^{-\int_{t^{\prime}}^{t} \frac{1}{\tau}} .
\end{aligned}
$$

Under the hypothese (9) and using again $\int_{t^{\prime}}^{t^{\prime \prime}} \frac{1}{\tau} \leq \frac{\|h\|_{a}}{\lim \inf \tau}$, we get (10).

To get (8), we deduce from the previous inequality:

$$
\begin{aligned}
\liminf \zeta+k_{P}+x(t) & \leq\left(\min \left\{k_{P}+\lim \inf \zeta-\liminf \xi, 2 k_{P}\right\}+2 k_{P}\left(e^{\int_{t^{\prime}}^{t^{\prime \prime}} \frac{1}{\tau}}-1\right)\right) e^{-\int_{t^{\prime}}^{t} \frac{1}{\tau}} \\
& \leq \min \left\{\left(k_{P}+\lim \inf \zeta-\liminf \xi\right)^{+}, 2 k_{P}\right\} e^{-\int_{t^{\prime}}^{t^{\prime \prime}} \frac{1}{\tau}}+2 k_{P}\left(1-e^{-\int_{t^{\prime}}^{t^{\prime \prime}} \frac{1}{\tau}}\right)
\end{aligned}
$$

INRIA 
remarking that the largest value of the right-hand side is attained for $t=t^{\prime \prime}$. We then use the bound on $\int_{t^{\prime}}^{t^{\prime \prime}} \frac{1}{\tau}$.

Proof of Corollary 7. With the proposed choice for the control parameters, one gets, using the notations of Theorem 6

$$
\begin{aligned}
& K_{1}=\frac{1}{\liminf \tau}\left(k_{I}\|\tau\|_{a}+2 \delta_{\zeta}\right), \\
& K_{2}=k_{I}|\tau|_{a}+\delta_{\zeta}, \\
& K_{3}=-e^{-\frac{\|h\| a}{\lim \inf \tau}} K_{2} .
\end{aligned}
$$

We then have

$$
\tilde{T}=2 \lim \inf \tau \log \left(2 \frac{k_{I}|\tau|_{a}+\delta_{\zeta}}{k_{I} \lim \inf \tau}\right),
$$

so $\tilde{T}<0$, due to the bounds on $k_{I}$. Writing that

$$
K_{2}-K_{3}^{-} e^{-\frac{\hat{\frac{T}{2}}-\|h\|_{a}}{\liminf \tau}}=K_{2}\left(1-e^{-\frac{\hat{T}}{2 \lim \inf \tau}}\right) \leq K_{2} \frac{\hat{T}}{2 \liminf \tau},
$$

we obtain that $\hat{T} \leq 2\|h\|_{a}$. Hence, we have

$$
|x|_{a} \leq \delta_{\xi}+2 \Phi\left(2\|h\|_{a}\right)=\delta_{\xi}+k_{I}\|h\|_{a}+2\left(K_{2}+K_{3}\right),
$$

and a similar formula for $\|x\|_{a}$. Using again the bounds on $k_{I}$, we get $|x|_{a} \leq \delta_{\xi}+2 k_{I}\|h\|_{a}$, and similarly for $\|x\|_{a}$.

\section{Proof of Theorem 2 when the initial condition has a finite number of zeros}

Let $x$ be a solution of (1) corresponding to an initial condition with a finite number of zeros.

\subsection{Evolution of the number of zeros}

Since the elements of $Z$ are the zeros of $x$ where $x$ changes sign, the slope of $x$ changes sign between two consecutive points of $Z$. Moreover, by equation (1), the sign of the slope of $x$ around $t \in Z$ is opposite to the sign of $x$ around $t-h$. On the one hand, this implies that there is a change of the sign of $x(t-h)$ (and then a point of $Z-h$ if $t \geq h$ ) between two consecutive points of $Z$. Hence $\left.x\right|_{(t-h, t]}$ has a finite number of zeros for $t \geq 0$ and $V$ is nondecreasing.

On the other hand, the sign of the slope of $x$ around $t \in Z$ is the same as that of the first point of $Z$ following $t-h$. Hence, $V$ is even. Since $V$ is nonincreasing with integer even values, $V$ converges in finite time towards some limit $2 n$. From now on, the proof essentially reduces to the study of discrete systems describing the evolution on each level $V \equiv 2 n, n \in \mathbb{N}$. For advanced studies on related subjects, see [6, 12]. See also [9].

$\mathrm{RR} \quad \mathrm{n}^{\circ} 3422$ 


\subsection{The levels $V \equiv 2 n, n \in \mathbb{N}-\{0\}$}

We consider the increasing unbounded sequence $t_{k}$ of elements of $Z$. Here, we prove that if $V\left(t_{k}\right)=2 n \neq 0$ for any $k$ greater than a certain $k_{0} \geq 0$, then $x$ is equal to the periodic solution given in Theorem 1 with period $T_{n}^{*}$ for $t \geq t_{k_{0}}$. To this end, let us define the simplex $\Delta_{n} \triangleq\left\{b \in \mathbb{R}^{2 n}: b_{j} \geq 0, \sum_{j=1}^{2 n} b_{j} \leq \bar{h}\right\}$, and the map $\Phi_{n}: \Delta_{n} \rightarrow \mathbb{R}^{2 n}$ by:

$$
b^{\prime}=\Phi_{n}(b) \text { where } b_{j}^{\prime}=b_{j+1} \text { for } j \leq 2 n-1, b_{2 n}^{\prime}=\log \left(2 e^{\bar{h}-\sum_{1}^{2 n} b_{j}}-1\right) .
$$

The set $\Delta_{n}$ and the map $\Phi_{n}$ are respectively the state-space and the flow associated with the evolution on the level $V \equiv 2 n$. In other words, defining, for $k \geq k_{0}$ :

$$
b^{k, n} \triangleq \frac{1}{\tau}\left(t_{k-2 n+j}-t_{k-2 n+j-1}\right)_{1 \leq j \leq 2 n},
$$

we have

$$
b^{k+1, n}=\Phi_{n}\left(b^{k, n}\right) .
$$

The map $\Phi_{n}$ has a unique fixed point $b_{n}^{*} \in \Delta_{n}$, defined by $\left(b_{n}^{*}\right)_{j}=\frac{\bar{T}_{n}^{*}}{2}, j=1, \ldots, 2 n$ and corresponding to the unique periodic solution at level $V \equiv 2 n$. We shall show that, for any $b \in \Delta_{n}$, the sequence ${ }^{2}\left[\Phi_{n}^{-1}\right]^{k}(b)$ exists $\left(\Delta_{n}\right.$ is $\Phi_{n}^{-1}$-stable) and tends to $b_{n}^{*}$ when $k \rightarrow+\infty$. To this end, remark that

$$
\Phi_{n}^{-1}(b)-\Phi_{n}^{-1}\left(b^{\prime}\right)=\left(\int_{0}^{1} \nabla \Phi_{n}^{-1}\left(b^{\prime}+s\left(b-b^{\prime}\right)\right) \cdot d s\right)\left(b-b^{\prime}\right) .
$$

Now, for any $b \in \Delta_{n}$, one has $\nabla \Phi_{n}^{-1}(b) \in \mathcal{M}$, where $\mathcal{M} \triangleq\left\{M \in \mathbb{R}^{2 n \times 2 n} \quad: \quad M_{j+1, j}=\right.$ $1, M_{1, j}=-1$ for $j=1, \ldots, 2 n-1,-\frac{1}{1+e^{-\bar{h}}} \leq M_{1,2 n} \leq-\frac{1}{2}, M_{j, j^{\prime}}=0$ otherwise $\}$. So we deduce, since $\mathcal{M}$ and $\Delta_{\infty}$ are convex sets:

$$
\forall b, b^{\prime} \in \Delta_{n}, \exists M \in \mathcal{M}, \Phi_{n}^{-1}(b)-\Phi_{n}^{-1}\left(b^{\prime}\right)=M\left(b-b^{\prime}\right) .
$$

We are now led to the demonstration of an absolute stability property for a class of discrete dynamical systems. Defining a norm in $\mathbb{R}^{2 n}$ by $\|b\| \triangleq \sum_{j}\left|b_{j}\right|+\left|\sum_{j} b_{j}\right|$, one shows the following result:

Lemma 9. The following claims are true:

- $\forall M \in \mathcal{M}, \forall b \in \mathbb{R}^{2 n},\|M b\| \leq\|b\|$.

- $\forall M^{(k)} \in \mathcal{M}, k=1, \ldots, 2 n, \forall b \in \mathbb{R}^{2 n},\left\|\prod_{k=1}^{2 n} M^{(k)} b\right\|=\|b\|$ implies $b_{j} b_{j+1} \geq 0$ for $j=1, \ldots, 2 n-1$.

${ }^{2}$ Here, $\left[\Phi_{n}^{-1}\right]^{k}=\left[\Phi_{n}^{-1}\right]^{k-1} \circ \Phi_{n}^{-1}, k \in \mathbb{N}-\{0\}$, where o denotes the composition. 
- $\forall m=1, \ldots, 2 n-1, \forall M^{(k)} \in \mathcal{M}, k=1, \ldots, 2 n m, \forall b \in \mathbb{R}^{2 n},\left\|\prod_{k=1}^{2 n m} M^{(k)} b\right\|=\|b\|$ implies $b_{j} b_{j+m} \geq 0$ for $j=1, \ldots, 2 n-m$.

- $\forall b \in \mathbb{R}^{2 n}, \forall M^{(k)} \in \mathcal{M}, k=1, \ldots, 2 n(2 n-1)+1,\left\|\prod_{k=1}^{2 n(2 n-1)+1} M^{(k)} b\right\|=\|b\| \Rightarrow b=0$.

From the last property and compacity of $\mathcal{M}$, we deduce

$$
\sup _{\substack{M^{(k)} \in \mathcal{M} \\ k=1, \ldots, 2 n(2 n-1)+1}}\left\|\prod_{k=1}^{2 n(2 n-1)+1} M^{(k)}\right\|<1 .
$$

Then, $\left[\Phi_{n}^{-1}\right]^{2 n(2 n-1)+1}$ is a contraction on $\Delta_{n}$. Since by hypothesis $b^{k, n}=\left[\Phi_{n}\right]^{k-k_{0}}\left(b^{k_{0}, n}\right) \in$ $\Delta_{n}, k \geq k_{0}$, and $\Delta_{n}$ is bounded, then $b^{k_{0}, n}=b_{n}^{*}$. From this, we deduce that $x(t)=$ $x_{n}^{*}\left(t-h-t_{k_{0}}\right)$ or $x(t)=x_{n}^{*}\left(t-h-t_{k_{0}}-\frac{T_{n}^{*}}{2}\right)$ for $t \geq t_{k_{0}}$.

It remains to prove Lemma 9:

Proof of Lemma 9. - The first point is deduced by the fact that $M$ has the following form:

$$
M b=\left(\begin{array}{c}
-\sum_{j=1}^{2 n-1} b_{j}+M_{1,2 n} b_{2 n} \\
b_{1} \\
\cdots \\
b_{2 n-1}
\end{array}\right),
$$

and $-1 \leq M_{1,2 n} \leq 0$.

- Defining for the second point, $M=\prod_{k=1}^{2 n} M^{(k)}$ (where the product is taken from left to right), we deduce:

$$
M b=\left(\begin{array}{c}
\left(1+M_{1,2 n}^{(1)}\right) b_{1}-M_{1,2 n}^{(2)} b_{2} \\
\cdots \\
\left(1+M_{1,2 n}^{(2 n-1)}\right) b_{2 n-1}-M_{1,2 n}^{(2 n)} b_{2 n} \\
-\sum_{j=1}^{2 n-1} b_{j}+M_{1,2 n}^{(2 n)} b_{2 n}
\end{array}\right)
$$

$\mathrm{RR} \mathrm{n}^{\circ} 3422$ 
and (recall that $\left.-1<M_{1,2 n}^{(j)}<0\right)$ :

$$
\begin{aligned}
\|M b\| & =\sum_{j=1}^{2 n-1}\left|\left(1+M_{1,2 n}^{(j)}\right) b_{j}-M_{1,2 n}^{(j+1)} b_{j+1}\right|+\left|\sum_{j=1}^{2 n-1} b_{j}-M_{1,2 n}^{(2 n)} b_{2 n}\right|-M_{1,2 n}^{(1)}\left|b_{1}\right| \\
\leq & \sum_{j=1}^{2 n-1}\left(\left(1+M_{1,2 n}^{(j)}\right)\left|b_{j}\right|-M_{1,2 n}^{(j+1)}\left|b_{j+1}\right|\right)+\left|\sum_{j=1}^{2 n-1} b_{j}-M_{1,2 n}^{(2 n)} b_{2 n}\right|-M_{1,2 n}^{(1)}\left|b_{1}\right| \\
& \left.\quad \text { with equality if and only if } b_{j} b_{j+1} \geq 0, j=1, \ldots, 2 n-1\right) \\
& =\sum_{j=1}^{2 n-1}\left|b_{j}\right|-M_{1,2 n}^{(2 n)}\left|b_{2 n}\right|+\left|\sum_{j=1}^{2 n-1} b_{j}-M_{1,2 n}^{(2 n)} b_{2 n}\right| \\
\leq & \|b\| .
\end{aligned}
$$

- The third point is proved by induction on $m$. For instance, for $m=2$,

$$
\left\|\prod_{k=1}^{4 n} M^{(k)} b\right\| \leq\left\|\prod_{k=2 n+1}^{4 n} M^{(k)} b\right\| \leq\|b\|,
$$

and, if the extremal expressions are equal, then, by second point,

$$
b_{j} b_{j+1} \geq 0 \text { and }(M b)_{j}(M b)_{j+1} \geq 0, \forall j=1, \ldots, 2 n-1
$$

where $M b=\prod_{k=2 n+1}^{4 n} M^{(k)} b$. Using the expression of $(M b)_{j}$ as in (19), and the fact that $-1<M_{1,2 n}^{(j)}<0$, we deduce that $b_{j} b_{j+2} \geq 0, j=1, \ldots, 2 n-2$.

- For the last point, we apply the third one to $b$ and $M b$, where $M=M^{(2 n(2 n-1)+1)}$. We then obtain that all the coefficients of $b$ (resp. $M b$ ) have same sign. If $b \neq 0$, then for instance $b_{j} \geq 0$ for any $j=1, \ldots, 2 n$. Hence, $(M b)_{1}<0\left(\right.$ as $\left.M_{1,2 n}<0\right)$ and $(M b)_{j}=b_{j-1} \leq 0$ for any $j=2, \ldots, 2 n$. This implies $b_{1}=\cdots=b_{2 n-1}=0, b_{2 n}>0,(M b)_{1}=M_{1,2 n} b_{2 n}<0$, and $\|M b\|<\|b\|$, which contradicts the hypotheses.

\subsection{The level 0}

It remains to prove that any evolution at level $V \equiv 0$ tends in a finite time towards the SO cycle. Indeed, this is clear, as this happens as soon as $V(t)=0$ : at that instant, $x$ crosses zero and the evolution is the slowly oscillating periodic one, see also [22, 9].

Remark that when (PI) holds, the function $V$ does not decrease anymore. Indeed, it is possible to show, as in $[18,10]$ that the integer part of $\frac{V+1}{2}$ decreases. 


\section{Proof of Theorem 2 when the initial condition has an infinite number of zeros}

Consider now a solution $x$ of (1) corresponding to an initial condition with infinite number of zeros. In order to prove that $V(t)$ is finite after a finite time, we follow the technique of [17], except that we consider the set $Z$ of zeros with change of sign instead of the set of all zeros. This is possible because of our choice of sgn0, which has as a consequence that the set of all zeros of $x$ on $[0,+\infty)$ is zero-measured (see Theorem 5 above).

\subsection{Properties of the set $Z$}

Lemma 10. The set $Z$ is closed.

Proof. Let $t_{i}$ be a sequence of elements of $Z$ converging to $t$. There exists a subsequence, also denoted $t_{i}$, which converges e.g. from below. Let $t_{i}^{\prime}<t_{i}<t_{i}^{\prime \prime}<t$ be such that $x\left(t_{i}^{\prime}\right) x\left(t_{i}^{\prime \prime}\right)<0$, together with $t_{i}^{\prime \prime}-t_{i}^{\prime} \rightarrow 0$ (see (4)). Then $t_{i}^{\prime}, t_{i}^{\prime \prime}$ tend to $t$.

Let $\varepsilon>0$ be fixed. There exists $i$ such that $t_{i}^{\prime}, t_{i}^{\prime \prime} \in(t-\varepsilon, t)$. Now, as is proved in Theorem 5 above, the set of all zeros of $x$ on $[0,+\infty)$ is zero-measured, due to the choice of sgn0. Hence, there exists $t^{\prime \prime} \in(t, t+\varepsilon)$ such that $x\left(t^{\prime \prime}\right) \neq 0$. We have $x\left(t_{i}^{\prime}\right) x\left(t^{\prime \prime}\right)<0$ or $x\left(t_{i}^{\prime \prime}\right) x\left(t^{\prime \prime}\right)<0$, which proves that $t \in Z$ and completes the proof.

Let Acc $Z$ be the set of accumulation points of $Z$ (included in $Z$ by Lemma 10). The following result is also proved in [17].

Lemma 11. If $t \in \operatorname{Acc} Z, t \geq h$, then $t-h \in \operatorname{Acc} Z$.

Proof. Indeed, if $t \in \operatorname{Acc} Z$, then there exists for instance an increasing sequence $t_{i} \in Z$ with $x$ increasing around the points $t_{2 i}$ and decreasing around the points $t_{2 i+1}$. Then, $\operatorname{sgn} x\left(t_{i}-h\right)=(-1)^{i+1}$, and there exists $t_{i}^{\prime} \in\left(t_{i}, t_{i+1}\right)$ such that $t_{i}^{\prime}-h \in Z$. Hence, $t-h=\lim _{n \rightarrow+\infty} t_{i}^{\prime}-h \in \operatorname{Acc} Z$.

\subsection{Principle of the proof}

Let $t \geq 2 h$ be such that $V(t)=+\infty$. Suppose first that $[0, t] \cap Z=\emptyset$ or $t^{\prime}=\sup [0, t] \cap Z<h$, then, by Theorem 5, we have e.g. $x>0$ almost everywhere on $[h, t]$. Hence, $x$ decreases from time $t$ to a certain time $t^{\prime \prime}$ where $x$ crosses 0 and changes sign. Then, $V\left(t^{\prime \prime}\right)=0$, and the evolution is the slowly oscillating one. Indeed, if $[0, t] \cap Z \neq \emptyset$, then the proof of Theorem 6 implies that $t^{\prime \prime}-t^{\prime} \leq \frac{T_{0}^{*}}{2}$ where $T_{0}^{*}$ is defined by (3), so $t^{\prime \prime} \leq h+\frac{T_{0}^{*}}{2}$. If $[0, t] \cap Z=\emptyset$, the value of $t^{\prime \prime}$ depends necessarily upon the initial condition $x_{0}$. Suppose now that $t^{\prime} \geq h$. Therefore, by the definition of $V$, there exists an infinite number of elements of $Z$ in $\left[t^{\prime}-h, t^{\prime}\right]$. Then by compacity, there exists at least one accumulation point $\alpha$ of $Z$ in $\left[t^{\prime}-h, t^{\prime}\right]$. Hence, for any $t \geq 2 h$ such that $V(t)=+\infty$ and $t^{\prime} \geq t-h \geq h$, there exists $\alpha \in \operatorname{Acc} Z$ with $\alpha \geq t-2 h$. Conversely, if $\alpha \in \operatorname{Acc} Z$ and $\alpha \geq h, V\left(\alpha+\frac{h}{2}\right)=+\infty$. Hence, $V(t)$ is finite after a finite time if and only if Acc $Z$ is empty or bounded. If Acc $Z \neq \emptyset$ is bounded, $V(t)$ is finite for 
$t>\max \operatorname{Acc} Z+h+\frac{T_{0}^{*}}{2}$ (by the same arguments as for the bound on $t^{\prime \prime}$ ). We prove below that when Acc $Z$ is non-empty, it is bounded, and give a bound for $\max A c c Z$ in function of the initial condition $\left.x\right|_{(-h, 0]}$.

For this purpose, we fix $\alpha \in \operatorname{Acc} Z, \alpha \geq 2 h(\alpha$ large if $\sup \operatorname{Acc} Z=+\infty, \alpha=\max \operatorname{Acc} Z$ otherwise). By Lemma 11, $\alpha-k h \in \operatorname{Acc} Z$ for all $k \in \mathbb{N}, k \leq \frac{\alpha}{h}$. Then, we construct a function $\nu$ of $\alpha \in \operatorname{Acc} Z, \alpha \geq h$ (depending on $Z$ ), such that on the one hand $\nu(\alpha-k h)$ decreases and "tends to 0 when $k \rightarrow+\infty$ ", and on the other hand, $\nu\left(\alpha-k_{0} h\right)$ is lower bounded by some constant (depending on $\left.x\right|_{(-h, 0]}$ ), for $k_{0}=\left\lfloor\frac{\alpha}{h}\right\rfloor-1$. Here, $\left\lfloor\frac{\alpha}{h}\right\rfloor$ denotes the integer part of $\frac{\alpha}{h}$. This furnishes a bound for $k_{0}$ and then for $\alpha$.

Lemma 12. The following formula defines a nonnegative real-valued function on the set of bounded open sets $U$ of $\mathbb{R}$ :

$$
\mu(U) \triangleq \sum_{\begin{array}{c}
\text { I connected } \\
\text { component of } U
\end{array}} \log \cosh \frac{\text { meas } I}{2 \tau} .
$$

It has the properties:

$$
\left(\sup _{\begin{array}{c}
I \text { connected } \\
\text { component of } U
\end{array}} \operatorname{meas} I\right)^{2} \frac{\log \cosh \frac{\operatorname{meas} U}{2 \tau}}{(\operatorname{meas} U)^{2}} \leq \mu(U) \leq \frac{\operatorname{meas} U}{2 \tau}<+\infty .
$$

Proof. Any bounded open set $U$ of $\mathbb{R}$ has at most a countable number of connected components, and they are open intervals. Since $\log \cosh \frac{u}{2} \geq 0$ for all $u \geq 0$, the sum in (20) does not depend upon the ordering on the set of connected components, and $\mu$ is hence welldefined. It is a $\sigma$-additive positive function on the open sets, that is $\mu(U)=\sum_{i \in \mathcal{I}} \mu\left(U_{i}\right)$ if $\left\{U_{i}: i \in \mathcal{I} \subset \mathbb{N}\right\}$ is a partition of $U$ into open sets.

The right inequality in (21) follows from the property $\log \cosh \frac{u}{2} \leq \frac{u}{2}$ for all $u \geq 0$. The left one is due to the fact that $\log \cosh \left(\frac{u}{2 \tau}\right) / u^{2}$ decreases for $u \geq 0$.

Proposition 13. If (P) holds, then, for any solution of (1), the following formula defines a nonnegative real-valued function on the set of open intervals $I$ of $\mathbb{R}^{+}$with bounds in Acc $Z$ and length meas $I \leq h$ :

$$
\nu(I) \triangleq \mu(I \backslash Z) .
$$

Moreover, there exists $\theta>0$ such that, for any open interval $I \subset[h,+\infty)$ with bounds in Acc $Z$ and length meas $I \leq h$,

$$
\nu(I-h) \leq \nu(I)-\theta \tau^{4} \frac{\nu(I)^{3}}{(\operatorname{meas} I)^{4}},
$$

where by definition, $I-h$ denotes the set I translated by $-h$. 
For any open interval $I$ with bounds in Acc $Z$ and length meas $I \leq h$, the set $I \backslash Z$ is open, since $Z$ is closed, and such that meas $(I \backslash Z)=$ meas $I$, since meas $Z=0$ by Theorem 5 . Hence, the definition of $\nu$ in Proposition 13 is meaningful. The proof of (22) is the subject of Sections 5.3 to 5.6. We first end the proof of Theorem 2 by using Proposition 13.

Defining

$$
\nu(\alpha) \triangleq \nu((\alpha-h, \alpha)) \text { for } \alpha \in \operatorname{Acc} Z, \alpha \geq h,
$$

inequality (22) implies in particular that

$$
\nu(\alpha-h) \leq \nu(\alpha)-\theta \frac{\nu(\alpha)^{3}}{\bar{h}^{4}},
$$

and then (see [17, Proposition 2.1]):

$$
\nu(\alpha-k h) \leq \frac{\bar{h}^{2}}{\sqrt{(2 k+1) \theta}} \text { for } k \leq k_{0} \triangleq\left\lfloor\frac{\alpha}{h}\right\rfloor-1 .
$$

From (21), we get

$$
\nu\left(\alpha-k_{0} h\right) \geq\left(\sup _{i \in \mathcal{I}} l_{i}\right)^{2} \frac{\log \cosh \frac{\bar{h}}{2}}{h^{2}},
$$

where $l_{i}, i \in \mathcal{I} \subset \mathbb{N}$ are the lengths of the connected components of $\left(\alpha-\left(k_{0}+1\right) h, \alpha-k_{0} h\right) \backslash Z$. By the definition of $Z$, the connected components of $Z^{\mathrm{c}}=\{t \geq 0: t \notin Z\}$ are the maximal intervals where $x$ has a constant sign a.e. Let $I$ be an interval of $(-h,+\infty)$ with e.g. $\operatorname{sgn} x(t)=1$ a.e. on $I$. Then, $\tau \dot{x}+x=-1$ a.e. on $I+h$, and $x$ decreases in $I+h$, which implies that either $x$ has constant sign on $I+h$, or $x$ is positive on the first part of the interval $I+h$ and negative on the other part. Hence, $I+h$ is included in the union of the closure of two connected components of $Z^{\mathrm{c}}$. This implies that:

$$
\begin{aligned}
\delta & \triangleq \sup \{\operatorname{meas} I: I \subset(-h, 0), \operatorname{sgn} x \text { constant a.e. on } I\} \\
& \leq 2 \sup \{\operatorname{meas} I: I \text { connected component of }(0, h) \backslash Z\} \\
& \leq 4 \sup _{j \in \mathcal{J}} l_{j} .
\end{aligned}
$$

The last inequality is deduced from the fact that $\left(\alpha-\left(k_{0}+1\right) h, \alpha-k_{0} h\right) \subset(0,2 h)$. Therefore,

$$
\nu\left(\alpha-k_{0} h\right) \geq \frac{\log \cosh \frac{\bar{h}}{2}}{16}\left(\frac{\delta}{h}\right)^{2},
$$

and, by (23):

$$
k_{0} \leq \frac{1}{2}\left(\frac{1}{\theta}\left(\frac{16 \bar{h}^{2}}{\log \cosh \frac{h}{2}}\right)^{2}\left(\frac{\delta}{h}\right)^{-4}-1\right)
$$

$\mathrm{RR} \mathrm{n}^{\circ} 3422$ 
This implies:

$$
\max \operatorname{Acc} Z \leq \tau c\left(1+\left(\frac{\delta}{h}\right)^{-4}\right),
$$

with $\delta$ defined in (24) and $c$ depending on $\bar{h}$. This achieves the proof of Theorem 2 (see the beginning of the present section).

It now remains to prove formula (22). This is made in the remaining of Section 5.

\subsection{Proof of (22) in a particular case where $I$ and $I-h$ do not contain accumulation points of $Z$}

If $I$ is an open interval with bounds in $\operatorname{Acc} Z$ such that $I \cap \operatorname{Acc} Z=\emptyset$, then the points of $Z \cap I$ may be ordered in increasing order. Therefore, the connected components of $U \triangleq I \backslash Z$ may be ordered in increasing order too. Let us denote them by $U_{j}, j \in \mathcal{J} \subset \mathbb{Z}\left(U_{j}\right.$ increasing wrt $j$ ). Let us define the sequence $b$ by: $b_{j} \triangleq$ meas $U_{j} / \tau$ if $j \in \mathcal{J}, b_{j} \triangleq 0$ otherwise. We have meas $U=\tau \sum_{j \in \mathbb{Z}} b_{j}$ and $\nu(I)=\mu(b) \triangleq \sum_{j \in \mathbb{Z}} \log \cosh \frac{b_{j}}{2}$. The sequence $b$ belongs to the following set:

$$
\Delta_{\infty} \triangleq\left\{\left(b_{j}\right)_{j \in \mathbb{Z}}: b_{j} \geq 0,0<\sum_{j \in \mathbb{Z}} b_{j} \leq \bar{h}, b_{j}=0 \Rightarrow b_{j^{\prime}}=0 \text { for } j^{\prime} \leq j \text { or } b_{j^{\prime}}=0 \text { for } j^{\prime} \geq j\right\} .
$$

Since, by the definition of $Z$, the sign of $x$ is constant a.e. in $U_{j}$ and alternating wrt $j$, we may impose $\operatorname{sgn} x \equiv(-1)^{j}$ a.e. on $U_{j}$.

Suppose now that $I-h$ has also no accumulating points of $Z$ inside. Denote $\tilde{U}_{j}$ the connected components of $\tilde{U} \triangleq I \backslash(Z+h)$, increasingly ordered as the $U_{j}$ 's, and denote $\tilde{b}_{j} \triangleq$ meas $\tilde{U}_{j} / \tau$. Since $x$ increases (resp. decreases) around $t \in I$ if and only if $x(.-h)<0$ (resp. $x(.-h)>0$ ) a.e. around $t$, the sets $\tilde{U}_{j}$ are exactly the connected components of the open subset of $I$ containing all the points around which $x$ is monotonous. The sequence $\tilde{b} \triangleq\left(\tilde{b}_{j}\right)_{j \in \mathbb{Z}} \in \Delta_{\infty}$ verifies the following property: there exists $\tilde{x} \in W^{1,+\infty}(I)$ such that, for all $j \in \mathbb{Z}$,

$$
\tau \dot{\tilde{x}}+\tilde{x}=(-1)^{j-1} k_{P} \text { a.e. on } \tilde{U}_{j},(-1)^{j} \tilde{x}>0 \text { a.e. on } U_{j} .
$$

Any element of $\Delta_{\infty}$ verifying this property is called a predecessor of $b$. A particularly simple predecessor, denoted $b^{\prime}$, is obtained by using a function $\tilde{x}$ satisfying (25) and being either increasing/decreasing, or decreasing/increasing, on any interval $U_{j}$. We denote $x^{\prime}$ the corresponding value of $\tilde{x}$. One shows easily (see Figure 5 ) that this corresponds to choose

$$
b_{j}^{\prime}=b_{j+1}+\log \left(1+e^{-b_{j+1}}\right)-\log \left(1+e^{-b_{j}}\right) .
$$

INRIA 


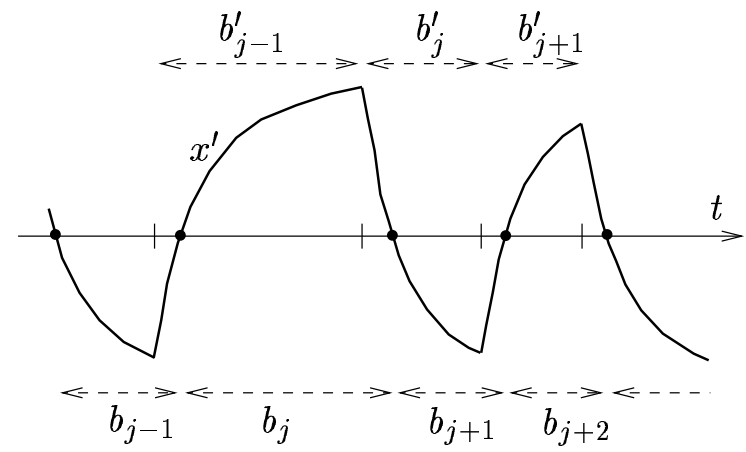

Figure 5: Optimal predecessor (The indexation shown corresponds to an arbitrary even integer $j$ )

This predecessor is called optimal for reasons which will become clear in 5.4 and 5.5.

Let us prove (22) in the particular case where $\tilde{b}=b^{\prime}$. We have:

$$
\begin{aligned}
\mu\left(b^{\prime}\right) & =\sum_{j \in \mathbb{Z}} \log \cosh \frac{b_{j}^{\prime}}{2} \\
& =\sum_{j \in \mathbb{Z}} \log \frac{e^{b_{j}^{\prime}}+1}{2}-\frac{1}{2} \sum_{j \in \mathbb{Z}} b_{j}^{\prime} \\
& =\sum_{j \in \mathbb{Z}} \log \left(\frac{1}{2}\left(\frac{1+e^{b_{j}}}{1+e^{-b_{j-1}}}+1\right)\right)-\frac{1}{2} \sum_{j \in \mathbb{Z}} b_{j} \quad \text { (due to the definition of } b^{\prime} \\
& \text { and the fact that } \left.\sum_{j \in \mathbb{Z}} b_{j}^{\prime}=\frac{\text { meas } \tilde{U}}{\tau}=\frac{\text { meas } I}{\tau}=\frac{\text { meas } U}{\tau}=\sum_{j \in \mathbb{Z}} b_{j}\right) \\
= & \sum_{j \in \mathbb{Z}} \log \frac{e^{b_{j}}+1}{2}-\frac{1}{2} \sum_{j \in \mathbb{Z}} b_{j}+\sum_{j \in \mathbb{Z}} \log \left(1+\frac{1}{1+e^{-b_{j-1}}}-\frac{1}{1+e^{-b_{j}}}\right) \\
\mu\left(b^{\prime}\right)= & \mu(b)+\sum_{j \in \mathbb{Z}} \log \left(1+\frac{1}{1+e^{-b_{j-1}}}-\frac{1}{1+e^{-b_{j}}}\right) .
\end{aligned}
$$

We now regroup some useful technical results in the following lemma, whose proof is elementary.

$\mathrm{RR} \mathrm{n}^{\circ} 3422$ 
Lemma 14. The following claims are true:

$$
\begin{gathered}
|u| \leq \frac{1}{1+e^{-\bar{h}}} \Rightarrow \log (1+u) \leq u-\theta_{1} u^{2} \\
\theta_{1} \triangleq\left(1+e^{-\bar{h}}\right)\left(1-\left(1+e^{-\bar{h}}\right) \log \left(1+\frac{1}{1+e^{-\bar{h}}}\right)\right)>0, \\
0 \leq u, v \leq \bar{h} \Rightarrow\left|\frac{1}{1+e^{-u}}-\frac{1}{1+e^{-v}}\right| \geq \theta_{2}|u-v|, \quad \theta_{2} \triangleq \frac{e^{-\bar{h}}}{\left(1+e^{-\bar{h}}\right)^{2}}>0, \\
u \geq 0 \Rightarrow \log \cosh \frac{u}{2} \leq \frac{1}{\theta_{3}} u^{2}, \quad \theta_{3} \triangleq 8 .
\end{gathered}
$$

From (27), (28), (29), we deduce

$$
\begin{aligned}
\mu\left(b^{\prime}\right) & \leq \mu(b)+\sum_{j \in \mathbb{Z}}\left(\frac{1}{1+e^{-b_{j-1}}}-\frac{1}{1+e^{-b_{j}}}\right)-\theta_{1} \sum_{j \in \mathbb{Z}}\left(\frac{1}{1+e^{-b_{j-1}}}-\frac{1}{1+e^{-b_{j}}}\right)^{2} \\
& \leq \mu(b)-\theta_{1} \theta_{2}^{2} \sum_{j \in \mathbb{Z}}\left(b_{j}-b_{j-1}\right)^{2} .
\end{aligned}
$$

Now, as $b$ is a nonnegative summable sequence, [17, Formulas (2.6) and (2.14)] implies that

$$
\left(\sum_{j \in \mathbb{Z}} b_{j}\right)^{4} \sum_{j \in \mathbb{Z}}\left(b_{j}-b_{j-1}\right)^{2} \geq 2\left(\sum_{j \in \mathbb{Z}} b_{j}^{2}\right)^{3} .
$$

We hence deduce, with the help of (30):

$$
\mu\left(b^{\prime}\right) \leq \mu(b)-\theta \frac{\mu(b)^{3}}{\left(\sum_{j \in \mathbb{Z}} b_{j}\right)^{4}},
$$

with $\theta \triangleq 2 \theta_{1} \theta_{2}^{2} \theta_{3}^{3}>0$. Since $\nu(I-h)=\mu\left(b^{\prime}\right)$ and $\nu(I)=\mu(b)$, then (22) is proved.

\subsection{Proof of (22) for general $I$ such that $I$ and $I-h$ do not contain accumulation points of $Z$}

We study here the case where $I \cap \operatorname{Acc} Z=I \cap(\operatorname{Acc} Z+h)=\emptyset$, so the connected components of $U \triangleq I \backslash Z$ and $\tilde{U} \triangleq I \backslash(Z+h)$ may be ordered as in Section 5.3, but we suppose that the predecessor $\tilde{b}$ of $b$ is different (up to a translation) from $b^{\prime}$ defined in (26). We prove that $\nu(I-h)=\mu(\tilde{b}) \leq \mu\left(b^{\prime}\right)$, which implies $(22)$, due to Section 5.3.

INRIA 


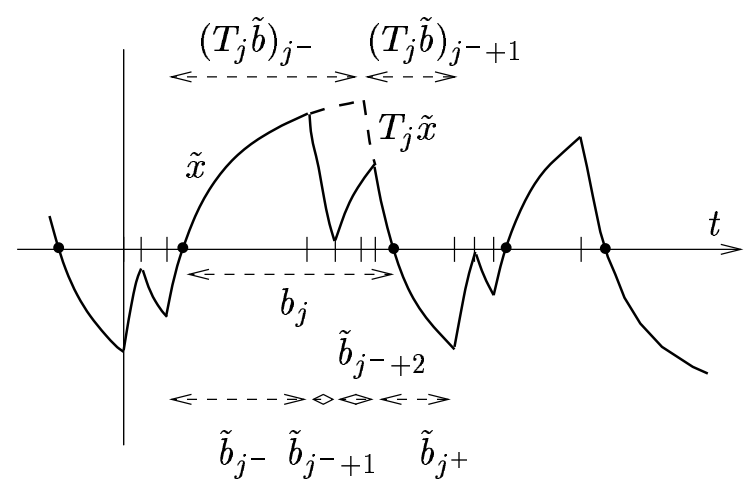

Figure 6: Suboptimal predecessors and their transformation $\left(j\right.$ and $j^{+}$are even, $p=$ $\left.\frac{j^{+}-j^{-}-1}{2}=1\right)$

Let $U_{j}, b_{j}, \tilde{U}_{j}, \tilde{b}_{j}, b_{j}^{\prime}, x^{\prime}$ be defined as in Section 5.3. The function $\tilde{x}=\left.x\right|_{I}$ satisfies (25).

For any $j \in \mathbb{Z}$, let $T_{j}$ be the following transformation on the function $\tilde{x}$ (see Figure 6 ):

$$
T_{j} \tilde{x}=\tilde{x} \text { on } I \backslash U_{j}, \quad T_{j} \tilde{x}=x^{\prime} \text { on } U_{j} .
$$

Let $j^{-}$(resp. $j^{+}$) be the unique index $j^{\prime}$ such that $\tilde{U}_{j^{\prime}}$ contains the left (resp. right) boundary of $U_{j}$. By the monotony of $\tilde{x}$ on the intervals $\tilde{U}_{j^{\prime}}$, we have $j^{+}-j^{-}=2 p+1$ with $p \in \mathbb{N}$ (Figure 6 shows a case where $p=1$ ). Moreover, $\tilde{U}_{j^{-+1}}, \ldots, \tilde{U}_{j^{+-1}} \subset U_{j}$. Let us partition $U_{j}$ into two subintervals $U_{j}^{-}$and $U_{j}^{+}$, with $U_{j}^{-} \leq U_{j}^{+}$and

$$
\frac{1}{\tau} \operatorname{meas} U_{j}^{-}=b_{j}^{-} \triangleq \log \left(\frac{1+e^{b_{j}}}{2}\right), \quad \frac{1}{\tau} \text { meas } U_{j}^{+}=b_{j}^{+} \triangleq b_{j}-b_{j}^{-}=-\log \left(\frac{1+e^{-b_{j}}}{2}\right) .
$$

If $\tilde{x}$ is equal to $x^{\prime}$ on $U_{j}$, then $p=0$ and

$$
\tilde{U}_{j^{-}} \cap U_{j}=U_{j}^{-}, \quad \tilde{U}_{j} \cap \cap U_{j}=U_{j}^{+} .
$$

The transformation $T_{j}$ generates the following transformation, also denoted $T_{j}$, on $\tilde{U}$ and $\tilde{b}$. The sets $\tilde{U}_{j^{-}}, \ldots, \tilde{U}_{j^{+}}$are replaced by $\left(T_{j} \tilde{U}\right)_{j^{-}}=\tilde{U}_{j^{-}} \cup U_{j}^{-}$and $\left(T_{j} \tilde{U}\right)_{j^{-}+1}=\tilde{U}_{j^{+}} \cup U_{j}^{+}$. Therefore, the numbers $\tilde{b}_{j^{-}}, \ldots, \tilde{b}_{j^{+}}$are replaced by $\left(T_{j} \tilde{b}\right)_{j^{-}}=b^{-}+b_{j}^{-}$and $\left(T_{j} \tilde{b}\right)_{j^{-+1}}=$ $b^{+}+b_{j}^{+}$, with

$$
b^{-} \triangleq \frac{\operatorname{meas} \tilde{U}_{j} \backslash U_{j}}{\tau}, \quad b^{+} \triangleq \frac{\operatorname{meas} \tilde{U}_{j+} \backslash U_{j}}{\tau} .
$$

The other components remain unchanged, up to a reindexation.

$\mathrm{RR} \mathrm{n}^{\circ} 3422$ 
Lemma 15. For all predecessor $\tilde{b}$ of $b$ and for all $j \in \mathbb{Z}$, we have

$$
\mu(\tilde{b}) \leq \mu\left(T_{j} \tilde{b}\right) .
$$

Proof. Let us consider the two $(2 p+2)$-dimensional vectors

$$
u \triangleq\left(\tilde{b}_{j^{-}}, \ldots, \tilde{b}_{j^{+}}\right), \quad v \triangleq\left(\left(T_{j} \tilde{b}\right)_{j^{-}},\left(T_{j} \tilde{b}\right)_{j^{-}+1}, 0, \ldots, 0\right) .
$$

Since $T_{j} \tilde{b}$ and $\tilde{b}$ differ only (up to a reindexation) by the coordinates present in $u$ and $v$, we have

$$
\mu\left(T_{j} \tilde{b}\right)-\mu(\tilde{b})=\sum_{l=1}^{2 p+2} \log \cosh \frac{v_{l}}{2}-\sum_{l=1}^{2 p+2} \log \cosh \frac{u_{l}}{2} .
$$

The proof of Lemma 15 then reduces to show $g(u) \leq g(v)$ for $g: \mathbb{R}^{2 p+2} \rightarrow \mathbb{R}, u \mapsto g(u)=$ $\sum_{l=1}^{2 p+2} \log \cosh \frac{u_{l}}{2}$. Since $\log \cosh$ is a convex function, $g$ is Schur-convex (see [15], [11], and [13, Proposition 3.C.1, p. 64], where a general presentation of the subject may be found). In other words, $g(u) \leq g(v)$ for any $u, v \in \mathbb{R}^{2 p+2}$ such that $u$ is majorized by $v$, that is fulfilling the two following conditions:

$$
\begin{gathered}
\sum_{l=1}^{2 p+2} u_{l}=\sum_{l=1}^{2 p+2} v_{l}, \\
\max _{\substack{\operatorname{card} \mathcal{L}=L \\
\mathcal{L} \subset\{1, \ldots, 2 p+2\}}} \sum_{l \in \mathcal{L}} u_{l} \leq \max _{\substack{\operatorname{card} \mathcal{L}=L \\
\mathcal{L}\{1, \ldots, 2 p+2\}}} \sum_{l \in \mathcal{L}} v_{l}, \quad \forall L=1, \ldots, 2 p+1 .
\end{gathered}
$$

It then suffices to prove that $v$ majorizes $u$, for $u, v$ given by (32).

Since

$$
\sum_{j^{\prime}=-\infty}^{+\infty} \tilde{b}_{j^{\prime}}=\sum_{j^{\prime}=-\infty}^{+\infty}\left(T_{j} \tilde{b}\right)_{j^{\prime}}=\sum_{j=-\infty}^{+\infty} b_{j}
$$

we have $\sum_{l=1}^{2 p+2} u_{l}=\sum_{l=1}^{2 p+2} v_{l}$, that is (33). Since $v$ has only two nonzero coordinates, (34) is fulfilled for $L \geq 2$. The case $L=1$ is equivalent to

$$
\max _{l=j^{-}, \ldots, j^{+}} \tilde{b}_{l} \leq \max \left\{\left(T_{j} \tilde{b}\right)_{j^{-}},\left(T_{j} \tilde{b}\right)_{j^{-+1}}\right\} .
$$

From the previous computations and as $b^{+}, b^{-} \geq 0$, it is sufficient to prove

$$
\max \left\{\tilde{b}_{j^{-}}-b^{-}, \tilde{b}_{j^{-}+1}, \ldots, \tilde{b}_{j^{+-1}}, \tilde{b}_{j^{+}}-b^{+}\right\} \leq \max \left\{b_{j}^{-}, b_{j}^{+}\right\},
$$


that is

$$
\max _{l=j^{-}, \ldots, j^{+}} \operatorname{meas} \tilde{U}_{l} \cap U_{j} \leq \max \left\{\operatorname{meas} U_{j}^{-}, \operatorname{meas} U_{j}^{+}\right\}
$$

Since the intervals $\tilde{U}_{l}$ are the maximal intervals on which $\tilde{x}$ is monotonous, the previous inequality means that the maximum length of an interval of monotony of $\tilde{x}$ in $U_{j}$ is less than that of $x^{\prime}$.

But it is easy to see from Figure 6 that the maximum length of a subinterval of $U_{j}$ on which $\tilde{x}$ increases is less than the length of the (unique) interval on which $x^{\prime}$ increases (that is $\tau b_{j}^{-}$if $j$ is even, $\tau b_{j}^{+}$if $j$ is odd). This is a consequence of the stationnarity of the equation $\tau \dot{x}+x=\varepsilon\left(\varepsilon= \pm k_{P}\right)$. Indeed, let us suppose that $j$ is even, and consider an interval $\left(t^{\prime}, t^{\prime \prime}\right) \subset U_{j}=\left(t, t+\tau b_{j}\right)$ where $\tilde{x}$ increases. Then $\tilde{x}$ satisfies $\tau \dot{\tilde{x}}+\tilde{x}=k_{P}$ on $\left(t^{\prime}, t^{\prime \prime}\right)$, and $\tilde{x}\left(t^{\prime}\right) \geq 0$. But $x^{\prime}$ satisfies the same equation on $\left(t, t+\tau b_{j}^{-}\right)$, and $x^{\prime}(t)=0$. If $t^{\prime \prime}-t^{\prime}>\tau b_{j}^{-}$, then $\tilde{x}\left(t^{\prime \prime}\right)>x^{\prime}\left(t+\tau b_{j}^{-}\right.$) (by the stationnarity of $\tau \dot{x}+x=k_{P}$ ), and $t^{\prime \prime}>t+\tau b_{j}^{-}$. Using all the properties of $x^{\prime}$ and $\tilde{x}$, that is: $x^{\prime}$ verifies $\tau \dot{x}^{\prime}+x^{\prime}=-k_{P}$ on $\left(t+\tau b_{j}^{-}, t+\tau b_{j}\right), \tilde{x}$ either increases or is a solution of the same equation on $\left(t^{\prime \prime}, t+\tau b_{j}\right), \tilde{x}\left(t^{\prime \prime}\right)>x^{\prime}\left(t+\tau b_{j}^{-}\right)$and $\tilde{x}\left(t+\tau b_{j}\right)=x^{\prime}\left(t+\tau b_{j}\right)=0$, we obtain that the trajectory of $\tilde{x}$ on $\left[t^{\prime \prime}, t+\tau b_{j}\right]$ contains all the trajectory of $x^{\prime}$ on $\left[t+\tau b_{j}^{-}, t+\tau b_{j}\right]$ but at different instants. This is impossible, since there is not enough time: $t+\tau b_{j}-t^{\prime \prime}<\left(t+\tau b_{j}\right)-\left(t+\tau b_{j}^{-}\right)$.

From this, we conclude that (recall that $j^{-}+2 p=j^{+}-1$ )

$$
\max \left\{\tilde{b}_{j^{-}}-b^{-}, \tilde{b}_{j^{-}+2}, \ldots, \tilde{b}_{j^{-}+2 p}\right\} \leq b_{j}^{-}
$$

Analogously, we may show that

$$
\max \left\{\tilde{b}_{j+-2 p}, \ldots, \tilde{b}_{j+-2}, \tilde{b}_{j+}-b^{+}\right\} \leq b_{j}^{+} .
$$

This leads to (35), and achieves the proof.

By applying the transformations $T_{j}$ successively for $j \in\{-m, \ldots, m\}$, we obtain a function $\tilde{x}^{m} \triangleq\left(T_{-m} \circ \cdots \circ T_{m}\right) \tilde{x}$ (the composition is commutative), equal to $x^{\prime}$ on the closure $\operatorname{cl}\left(\bigcup_{j=-m}^{m} U_{j}\right)$. Since the limit of the latter increasing sequence of sets is equal to $I, \tilde{x}^{m}$ tends to $x^{\prime}$ (pointwise). Applying the following lemma to $\tilde{b}^{m}=\left(T_{-m} \circ \cdots \circ T_{m}\right) \tilde{b}$, we obtain $\mu\left(\tilde{b}^{m}\right) \rightarrow \mu\left(b^{\prime}\right)$ when $m \rightarrow+\infty$, and finally deduce, with the help of Lemma 15 , that $\mu(\tilde{b}) \leq \mu\left(b^{\prime}\right)$. Therefore, $b^{\prime}$ is an optimal predecessor, in the sense that

$$
\mu\left(b^{\prime}\right)=\max _{\substack{\tilde{b} \in \Delta_{\infty} \\ \tilde{b} \text { predecessor of } b}} \mu(\tilde{b}),
$$

and (22) is proved for general $I$ such that $I$ and $I-h$ do not contain accumulation points of $Z$.

$\mathrm{RR} \mathrm{n}^{\circ} 3422$ 
Lemma 16. Let $\tilde{b}$ be a predecessor of $b$ and $\tilde{x}$ satisfy (25). If $\tilde{x}=x^{\prime}$ on the closure $\operatorname{cl}\left(\bigcup_{j=-m}^{m} U_{j}\right)$, then

$$
\left|\mu(\tilde{b})-\mu\left(b^{\prime}\right)\right| \leq \frac{1}{\tau} \operatorname{meas}\left(I \backslash \bigcup_{j=-m+1}^{m-1} U_{j}\right)=\sum_{j=-\infty}^{-m} b_{j}+\sum_{j=m}^{+\infty} b_{j} .
$$

Proof. The proof proceeds from the following inequalities:

$$
\begin{aligned}
\left|\mu(\tilde{b})-\mu\left(b^{\prime}\right)\right| \leq & \sum_{j \in \tilde{\mathcal{J}}} \log \cosh \frac{\tilde{b}_{j}}{2}+\sum_{j \in \mathcal{J}^{\prime}} \log \cosh \frac{b_{j}^{\prime}}{2} \\
& \text { where } \tilde{\mathcal{J}} \triangleq\left\{j \in \mathbb{Z}: \tilde{U}_{j} \not \subset \operatorname{cl}\left(\bigcup_{l=-m}^{m} U_{l}\right)\right\} \text { and similarly for } \mathcal{J}^{\prime} \\
\leq & \frac{1}{2} \sum_{j \in \tilde{\mathcal{J}}} \tilde{b}_{j}+\frac{1}{2} \sum_{j \in \mathcal{J}^{\prime}} b_{j}^{\prime} \\
\leq & \sum_{j=-\infty}^{-m} b_{j}+\sum_{j=m}^{+\infty} b_{j} \longrightarrow 0 \text { when } m \rightarrow+\infty,
\end{aligned}
$$

using the fact that $\log \cosh \frac{u}{2} \leq \frac{u}{2}$ for $u \geq 0$ and the definition of $\tilde{\mathcal{J}}, \mathcal{J}^{\prime}$.

\subsection{Proof of (22) for general $I$ such that $I$ does not contain accu- mulation points of $Z$}

We study here the case where $I \cap \operatorname{Acc} Z=\emptyset$, but $I \cap(\operatorname{Acc} Z+h) \neq \emptyset$. We use the same notations as in Section 5.4 for $U, \tilde{U}, U_{j}, U_{j}^{-}, U_{j}^{+}, b_{j}, b_{j}^{-}, b_{j}^{+}, b_{j}^{\prime}, \tilde{x}, x^{\prime}$. We prove again that $\nu(I-h)=\mu(\tilde{U}) \leq \mu\left(b^{\prime}\right)$, which implies $(22)$.

Since $I-h$ contains accumulation points of $Z$, the connected components $\tilde{U}_{i}, i \in \mathcal{I} \subset \mathbb{N}$ of $\tilde{U}$ cannot be ordered in increasing order; to recall this fact, we use a different notation of the indexes, namely $i \in \mathcal{I}$ instead of $j \in \mathcal{J}$. They still correspond to the intervals on which $\tilde{x}$ is monotonous. We define $T_{j}$ as in (31).

Contrarily to what happens in Section 5.4, for a given $j \in \mathcal{J}$, the number of intervals $\tilde{U}_{i}$ intersecting $U_{j}$ may be infinite, and these sets $\tilde{U}_{i}$ may possibly not be ordered in increasing order. Moreover, the boundaries of $U_{j}$ may be accumulation points of $Z+h$, and then of the (boundaries of the) intervals $\tilde{U}_{i}$. In this case, there may not exist an interval $\tilde{U}_{i}$ intersecting both $U_{j}$ and $U_{j-1}$ (resp. $U_{j+1}$ ), as $\tilde{U}_{j^{-}}$(resp. $\tilde{U}_{j^{+}}$) in Section 5.4.

Instead, we denote $j^{-}$(resp. $j^{+}$) the unique $i \in \mathcal{I}$, if it exists, such that the closure $\operatorname{cl}\left(\tilde{U}_{i}\right)$ contains the left (resp. right) boundary of $U_{j}$. We also denote $\mathcal{I}_{j} \triangleq\left\{i \in \mathcal{I} \backslash\left\{j^{-}, j^{+}\right\}\right.$: 
$\left.\tilde{U}_{i} \subset U_{j}\right\}$ and $\hat{\mathcal{I}}_{j} \triangleq \mathcal{I}_{j} \cup\left\{j^{-}, j^{+}\right\}$. If $j^{-}$(resp. $j^{+}$) does not exist, we put $\tilde{U}_{j^{-}}=\emptyset$ (resp. $\left.\tilde{U}_{j^{+}}=\emptyset\right)$.

The transformation of $\tilde{U}$ generated by $T_{j}$ acts as follows: the sets $\tilde{U}_{i}$ for $i \in \hat{\mathcal{I}}_{j}$ are replaced by $\left(T_{j} \tilde{U}\right)_{j^{-}}=\operatorname{conv}\left(\tilde{U}_{j^{-}} \cup U_{j}^{-}\right)$and $\left(T_{j} \tilde{U}\right)_{j^{+}}=\operatorname{conv}\left(\tilde{U}_{j^{+}} \cup U_{j}^{+}\right)$, where conv $S$ denotes the convex hull of the set $S$ (the convexification is needed to include the boundaries of $U_{j}$ ). The other components remain unchanged.

In order to achieve the proof, we generalize the results of Lemmas 15 and 16 .

Generalization of Lemma 16 is straightforward. It yields the implication

$$
\tilde{x}=x^{\prime} \text { on } \operatorname{cl}\left(\bigcup_{j=-m}^{m} U_{j}\right) \Rightarrow\left|\mu(\tilde{b})-\mu\left(b^{\prime}\right)\right| \leq \frac{1}{\tau} \operatorname{meas}\left(I \backslash \bigcup_{j=-m+1}^{m-1} U_{j}\right),
$$

which is proved as in the original form, replacing $\tilde{b}_{j}$ by meas $\tilde{U}_{i} / \tau$.

Generalization of Lemma 15 consists in proving that $\mu(\tilde{U}) \leq \mu\left(T_{j} \tilde{U}\right)$. This reduces to adapt the proof of Lemma 15 to the case $p=+\infty$. We consider the vectors $u, v \in \mathbb{R}^{\hat{\mathcal{I}}_{j}}$ defined by $u_{i}=$ meas $\tilde{U}_{i} / \tau$ for all $i \in \hat{\mathcal{I}}_{j}$ and $v_{i}=\operatorname{meas}\left(T_{j} \tilde{U}\right)_{i} / \tau$ for $i \in\left\{j^{-}, j^{+}\right\}, v_{i}=0$ otherwise. As in Lemma 15, we have to prove $g(u) \leq g(v)$, for $g: \mathbb{R}^{\hat{\mathcal{I}}_{j}} \rightarrow \mathbb{R}, u \mapsto \sum_{i \in \hat{\mathcal{I}}_{j}} \log \cosh \frac{u_{i}}{2}$.

Indeed, for the values of $g, u, v$ of Lemma 15 (that is for finite $p$ ), the inequality $g(u) \leq$ $g(v)$ holds under the only condition (34), since log cosh is increasing in $\mathbb{R}^{+}$and $u_{i}, v_{i} \geq 0$. The weaker order relation induced by (34) is called weak majorization, and the corresponding result is proved in $[19,21]$ and may be found in [13, 3.C.1.b, p. 64].

It is easy to check, by the same arguments as in Lemma 15 , that the vectors $u$ and $v$ satisfy the inequalities (34). Moreover, for any finite subset $\mathcal{S}$ of $\hat{\mathcal{I}}_{j}$ containing $j^{-}$and $j^{+}$, the truncatures $u_{\mathcal{S}}=\left(u_{i}, i \in \mathcal{S}\right), v_{\mathcal{S}}=\left(v_{i}, i \in \mathcal{S}\right)$ of $u, v$ still satisfy (34), that is here:

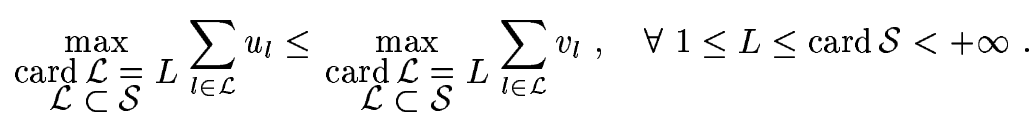

Then:

$$
\sum_{i \in \mathcal{S}} \log \cosh \frac{u_{i}}{2} \leq \sum_{i \in \mathcal{S}} \log \cosh \frac{v_{i}}{2}
$$

Passing to the limit when $\mathcal{S}$ tends towards $\hat{\mathcal{I}}_{j}$, we get $g(u) \leq g(v)$.

\subsection{Proof of (22) for general $I$}

Let $I$ be a general open interval with bounds in Acc $Z$ and meas $I \leq h$, and let $U=I \backslash$ Acc $Z$. The set $U$ is open, and is then the (finite or countable) union of its connected components $U_{i}, i \in \mathcal{I} \subset \mathbb{N}$, which form a partition of $U$. However in general, the sets $U_{i}$ cannot be 
anymore ordered (again, we use the same notation of the indexes). Since meas $Z=0$, we have:

$$
\sum_{i \in \mathcal{I}} \operatorname{meas} U_{i}=\operatorname{meas} U=\operatorname{meas} I
$$

Moreover, $I \backslash Z=U \backslash Z=\bigcup_{i \in \mathcal{I}} U_{i} \backslash Z$ (disjoint union) and, by the $\sigma$-additivity of $\mu$,

$$
\nu(I)=\sum_{i \in \mathcal{I}} \nu\left(U_{i}\right) .
$$

Since $t \in \operatorname{Acc} Z$ implies $t-h \in \operatorname{Acc} Z$ (by Lemma 11), then $(I-h) \backslash$ Acc $Z \subset U-h$ and $(I-h) \backslash Z=(U-h) \backslash Z=\bigcup_{i \in \mathcal{I}}\left(U_{i}-h\right) \backslash Z$ (disjoint union). Therefore,

$$
\nu(I-h)=\sum_{i \in \mathcal{I}} \nu\left(U_{i}-h\right) .
$$

Since (22) has already been proved for open intervals with bounds in Acc $Z$ and no accumulation points of $Z$ inside (see Section 5.5), we have, for any $i \in \mathcal{I}$ :

$$
\nu\left(U_{i}-h\right) \leq \nu\left(U_{i}\right)-\theta \tau^{4} \frac{\nu\left(U_{i}\right)^{3}}{\left(\operatorname{meas} U_{i}\right)^{4}} .
$$

Summing the previous inequalities, we obtain:

$$
\nu(I-h) \leq \nu(I)-\theta \tau^{4} \sum_{i \in \mathcal{I}} \frac{\nu\left(U_{i}\right)^{3}}{\left(\operatorname{meas} U_{i}\right)^{4}} .
$$

By use of Hölder inequality, we obtain:

$$
\begin{aligned}
\nu(I)=\sum_{i \in \mathcal{I}} \nu\left(U_{i}\right) & \leq\left(\sum_{i \in \mathcal{I}} \frac{\nu\left(U_{i}\right)^{3}}{\left(\operatorname{meas} U_{i}\right)^{4}}\right)^{\frac{1}{3}}\left(\sum_{i \in \mathcal{I}}\left(\operatorname{meas} U_{i}\right)^{2}\right)^{\frac{2}{3}} \\
& \leq\left(\sum_{i \in \mathcal{I}} \frac{\nu\left(U_{i}\right)^{3}}{\left(\operatorname{meas} U_{i}\right)^{4}}\right)^{\frac{1}{3}}(\text { meas } I)^{\frac{4}{3}} .
\end{aligned}
$$

Then,

$$
\nu(I-h) \leq \nu(I)-\theta \tau^{4} \frac{\nu(I)^{3}}{(\operatorname{meas} I)^{4}},
$$

and the proof of (22) is done for general $I$ with bounds in Acc $Z$.

INRIA 


\section{References}

[1] M. Akian, P.-A. Bliman, On super-high-frequencies in discontinuous 1st-order delaydifferential equations, in Proc. of 6th IEEE Mediterranean Conference on Control and Systems, Alghero (Italy), 9-11 June, 1998.

[2] M. Akian, P.-A. Bliman, On super-high-frequencies in discontinuous 1st-order delaydifferential equations, INRIA Research report, 1998.

[3] K. Aouchiche, P.-A. Bliman, M. Sorine, P.I. control of periodic oscillations of relay systems, Conference on Control of Oscillations and Chaos, St-Petersburg, Russia, August 27-29 (1997)

[4] K.J. Åström, T. Hägglund, Automatic tuning of simple regulators, Proc. of IFAC 9th World congress, Budapest (1984)

[5] K.J. Åström, B. Wittenmark, Adaptive control, Addison-Wesley Publishing Company (1989)

[6] O. Diekmann, S.A. van Gils, S.M. Verduyn Lunel, H.-O. Walther, Delay equations: functional-, complex- and nonlinear analysis, Applied Mathematical Sciences 110, Springer-Verlag, New-York, 1995

[7] G.F. Franklin, J.D. Powell, A. Emami-Naeini, Feedback control of dynamic systems, Addison-Wesley Publishing Company (1988)

[8] L. Fridman, E. Fridman, E. Shustin, Steady modes in an autonomous system with break and delay, Differential equations (translation of Differentsial'nye uravneniya), vol. 29, no $8,1161-1166$ (1993)

[9] L. Fridman, E. Shustin, E. Fridman, Steady modes and sliding modes in the relay control systems with time delay, Proc. 35th Conf. on Decision and Control, Kobe, Japan (1996), 4601-4606

[10] L. Fridman, E. Fridman, E. Shustin, The phenomenon of the second order steady modes in the relay control systems with time delay, Proc. of the 4 th European Control Conference, Brussels, Belgium (1997)

[11] G.H. Hardy, J.E. Littlewood, G. Pólya, Some simple inequalities satisfied by convex functions, Messenger Math. 58 (1929), 145-152

[12] J. Mallet-Paret, Morse decompositions for delay-differential equations, Journal of differential equations $\mathbf{7 2}$ (1988), 270-315

[13] A.W. Marshall, I. Olkin, Inequalities: theory of majorization and its applications, Academic Press, Serie Math. in Science and Engineeting 143 (1979)

$\mathrm{RR} \mathrm{n}^{\circ} 3422$ 
[14] R.D. Nussbaum, Periodic solutions of nonlinear autonomous functional differential equations, Functional differential equations and approximation of fixed points, Lecture Notes in Mathematics 730, Springer-Verlag (1979), 283-325

[15] I. Schur, Über eine Klasse von Mittelbildungen mit Anwendungen die Determinanten Theorie Sitzungsber. Berlin. Math. Gesellschaft 22 (1923), 9-20 (Isaie Schur collected works (A. Brauer and H. Rohrbach, eds.) Vol. II, Springer-Verlag, Berlin (1973), 416427)

[16] T. Seidman, The dynamics of some systems with hysteretic nonlinearities in Optimization and Nonlinear Analysis, (Ioffe, Marcus, Reich), Longman, Harlow (1992), 273-285

[17] E. Shustin, Super-high-frequency oscillations in a discontinuous dynamic system with time delay, Israel J. of Mathematics, 90 (1995), 199-219

[18] E. Shustin, E. Fridman, L. Fridman, Stable oscillations in a discontinuous delay system of the second order, Proc. Int. Conf. on Differential equations and dynamical systems, Springfield, Missouri, May 29-June 1 (1996)

[19] M. Tomić, Théorème de Gauss relatif au centre de gravité et son application, Bull. Soc. Math. Phys. Serbie 1 (1949), 31-40

[20] Ya.Z. Tsypkin, Relay control systems, Cambridge University Press (1984)

[21] H. Weyl, Inequalities between two kinds of eigenvalues of a linear transformation, Proc. Nat. Acad. Sci. U.S.A. 35 (1949), 408-411

[22] X. Xie, Uniqueness and stability of SOP solutions of delay equations with bounded nonlinearity, Journal of dynamics and differential equations $\mathbf{3}, \mathrm{n}^{\circ} 4$ (1991), 515-540 
Unit`e de recherche INRIA Lorraine, Technopôle de Nancy-Brabois, Campus scientifique, 615 rue du Jardin Botanique, BP 101, 54600 VILLERS LÈS NANCY

Unit'e de recherche INRIA Rennes, Irisa, Campus universitaire de Beaulieu, 35042 RENNES Cedex Unit'e de recherche INRIA Rhône-Alpes, 655, avenue de l'Europe, 38330 MONTBONNOT ST MARTIN Unit'e de recherche INRIA Rocquencourt, Domaine de Voluceau, Rocquencourt, BP 105, 78153 LE CHESNAY Cedex Unit'e de recherche INRIA Sophia-Antipolis, 2004 route des Lucioles, BP 93, 06902 SOPHIA-ANTIPOLIS Cedex

Éditeur

INRIA, Domaine de Voluceau, Rocquencourt, BP 105, 78153 LE CHESNAY Cedex (France) http://www.inria.fr ISSN 0249-6399 